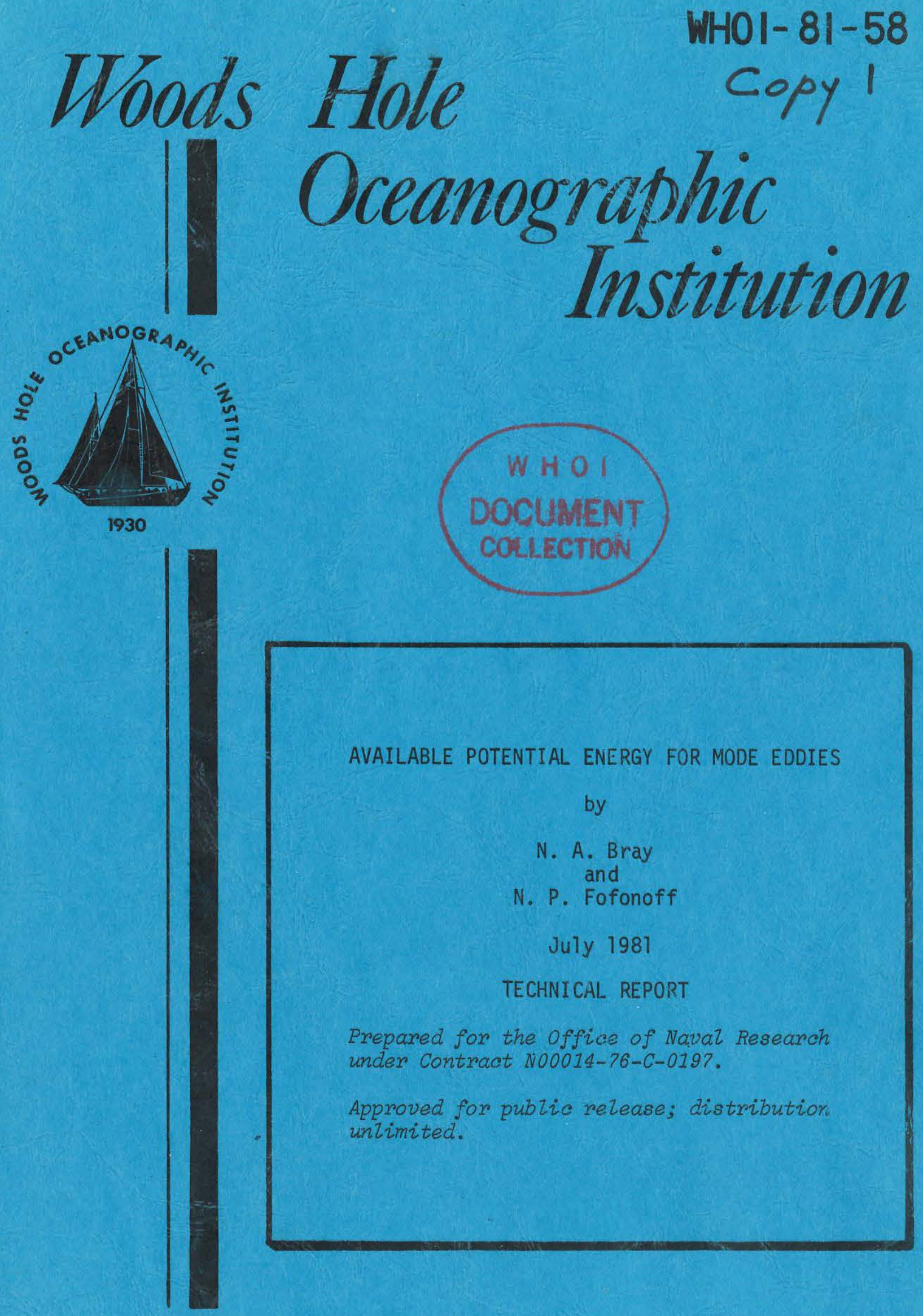

WOODS HOLE, MASSACHUSETTS 02543 
WHOI $-81-58$

AVAILABLE POTENTIAL ENERGY FOR MODE EDDIES

by

\author{
N. A. Bray \\ and \\ N. P. Fofonoff
}

WOODS HOLE OCEANOGRAPHIC INSTITUTION

Woods Hole, Massachusetts 02543

July 1981

TECHNICAL REPORT

Prepared for the Office of Naval Research under Contract N00014-76-C-0197.

Reproduction in whole or in part is permitted for any purpose of the United States Govermment. In citing this report in a bibliography, the reference given should be to: Journal of Physical Oceanography 2(1):: 30-47 (January 1981).

Approved for public release; distribution unlimited.

Approved for Distribution: Valentine Worthington, Chairman Department of Physical Oceanography 
Reprinted from Journal of Physical Oceanography, Vol. 11, No. 1, January 1981 American Meteorological Society

Printed in U. S. A.

\section{Available Potential Energy for MODE Eddies}

N. A. Bray and N. P. Fofonoff 


\title{
Available Potential Energy for MODE Eddies ${ }^{1}$
}

\author{
N. A. Bray and N. P. FofonofF \\ Woods Hole Oceanographic Institution, Woods Hole, MA 02543
}

(Manuscript received 14 November 1979, in final form 13 May 1980)

\begin{abstract}
Available potential energy (APE) is defined as the difference between total potential plus internal energy of a fluid in a gravity field and a corresponding reference field in which the fluid is redistributed (leveled) adiabatically to have constant stably-stratified densities along geopotential surfaces. Potential energy changes result from local shifts of fluid mass relative to geopotential surfaces that are accompanied by local changes of enthalpy and internal energy and global shifts of mass (because volumes of fluid elements are not conserved) that do not change enthalpy or internal energy. The potential energy changes are examined separately by computing available gravitational potential energy (GPE) per unit mass and total GPE (TGPE) per unit area.

A technique for estimating GPE in the ocean is developed by introducing a reference density field (or an equivalent specific volume anomaly field) that is a function of pressure only and is connected to the observed field by adiabatic vertical displacements. The full empirical equation of state for seawater is used in the computational algorithm. The accuracy of the estimate is limited by the data and sampling and not by the algorithm itself, which can be made as precise as desired.

The reference density field defined locally for an ocean region allows redefinition of dynamic height $\Delta D$ (potential energy per unit mass) relative to the reference field. TGPE per unit area becomes simply the horizontal average of dynamic height integrated over depth in the region considered. The reference density surfaces provide a precise approximation to material surfaces for tracing conservative variables such as salinity and potential temperature and for estimating vortex stretching between surfaces.

The procedure is applied to the MODE density data collected in 1973. For each group of stations within five 2-week time windows (designated Groups A-E) the estimated GPE is compared with the net APE based on the Boussinesq approximation and to the low-frequency kinetic energy measured from moored buoys. Changes of potential energy of the reference field from one time window to the next are large compared with the GPE within each window, indicating the presence of scales larger than the station grid.

An analysis of errors has been made to show the sensitivity of the estimates to data accuracy and sampling frequency.
\end{abstract}

\section{Introduction}

Nearly geostrophic flow in the ocean is maintained by potential energy stored as vertical displacements of density surfaces relative to level (geopotential) surfaces. If the rate of dissipation of kinetic energy is less than the conversion rate from potential energy, the flow accelerates or intensifies. If the conversion rate is less, the flow weakens. An initially unbalanced density field will tend to spin up toward a geostrophically balanced flow converting potential to kinetic energy by flow down pressure gradients (Saunders, 1973). An initially unbalanced velocity field will convert kinetic to potential energy to approach geostrophic balance (Rhines, 1977). Processes of geostrophic adjustment have received considerable attention in the literature starting with Rossby (1938).

The major conversion of potential to kinetic en-

\footnotetext{
${ }^{1}$ Contribution No. 4462 from the Woods Hole Oceanographic Institution. Mode Contribution No. 149.
}

ergy in the ocean occurs in western boundary currents such as the Gulf Stream. Potential energy accumulated in the ocean interior by heating and convergence of Ekman drift is converted to kinetic energy in the Gulf Stream as the flow is accelerated by the downstream pressure gradient. The conversion is partial because the constraints imposed by geostrophy require a strong cross-stream slope of density surfaces. Consequently, large quantities of both kinetic and potential energies are converted into the mesoscale range in the formation and breakdown regions of the Gulf Stream through a combination of barotropic and baroclinic instabilities (Rhines, 1977; Holland, 1978).

Significant conversion of potential energy may also occur to the mesoscale through baroclinic instability in open ocean regions where horizontal gradients of density are sufficiently strong (Gill et al., 1974; Robinson and McWilliams, 1974). Estimates from oceanographic data of the potential energy available for conversion to kinetic energy have not been used 
extensively in studying ocean dynamics. This may be due, in part, to the lack of an acceptably defined procedure for estimation. For small vertical displacements, the Boussinesq approximation for estimating available potential energy suffices. For large vertical displacements found in Gulf Stream rings, for example, this approximation cannot be expected to yield accurate estimates. Barrett (1971) and Cheney and Richardson (1976) used the anomaly of potential energy within the ring referenced to the surrounding region to evaluate available potential energy. Reid et al. (1981) point out that this method overestimates APE because replacement of a ring or eddy by the surrounding density field does not satisfy mass conservation and, therefore, includes a contribution to potential energy that biases the estimate.

Vulis and Monin (1975) estimated available potential energy for the North Atlantic by leveling the entropy field (computed from observed mean temperature, neglecting salinity variations) with respect to the pressure field. Their estimate of $700 \mathrm{~J} \mathrm{~m}^{-3}$ averaged over the volume of the Atlantic is of the same magnitude as the atmospheric available potential energy for the Northern Hemisphere calculated by Peixóto and Oort (1974). The error in neglecting salinity was not evaluated.

Lorenz (1955) introduced the concept of available potential energy (APE) in the study of energetics of the atmosphere. He pointed out that no conversion of potential to kinetic energy can occur if the density field is uniform on geopotential surfaces. Such a state represents a relative minimum of potential energy. If the stratification is stable, no further reduction of potential energy is possible by adiabatic processes. The potential energy, of course, can be reduced further by nonadiabatic processes such as heat and salt exchange through boundary fluxes and internal diffusion. Lorenz defined APE as the difference between the potential plus internal energy of the observed density field and a reference field that is obtained by redistributing mass adiabatically so that density is uniform on geopotential surfaces. Lorenz used the sum of internal and potential energy in his definition. The total internal energy is proportional to the potential energy assuming a perfect gas law for the atmosphere. Because proportionality between the total internal and potential energies does not hold for the ocean and because changes of internal energy have a different distribution within a water column than the potential energy changes, the potential energy changes are computed separately.

Following the terminology introduced by Reid et al. (1981), we will define the difference of potential energy per unit mass of the observed density field and the corresponding adiabatically leveled field as the available gravitational potential energy (GPE) and the vertical integral over the column as total available gravitational potential energy (TGPE). Thus, the net or "true" APE is the sum of TGPE and the internal energy change per unit area. The separation permits examination and comparison of relative magnitudes and vertical distribution within the water column that is not readily available from the integral expressions for APE or from the enthalpy formulation used by Reid et al. (1981).

The procedure for estimating GPE is applied to the hydrographic data set obtained during the Mid-Ocean Dynamics Experiment (MODE) carried out in 1973. About 800 vertical profiles of temperature and salinity were obtained with CTD and STD profiles during the 4-month duration of the experiment (Scarlet, $1975)$ to provide a unique description of the eddy field in space and time. The measurements do not permit an evaluation of the complete pressure field relative to geopotential surfaces. Hence, evaluation of GPE and APE are restricted to leveling with respect to the pressure surfaces rather than geopotential surfaces. The reference density field is a function of pressure only so that no conversion of potential energy to baroclinic motion can occur by adiabatic processes. Barotropic processes are not considered further.

The magnitude of APE is clearly dependent on the region under consideration. In Lorenz's definition the total potential energies were calculated for the entire atmospheric mass. In the present discussion, the calculations are made for a small region of the ocean (where data are available) to estimate local storage of APE. It is assumed that locally defined APE on the eddy scale is the appropriate estimate for local mesoscale eddies. An adequate evaluation of the appropriate choice of a region to define the reference field for proper separation of mesoscale and ocean scale contributions requires considerable additional research and is recognized but cannot be addressed in terms of the spatially limited MODE data.

The classical definitions of dynamic height and anomaly of potential energy (Sandstrom and Helland-Hansen, 1903; Fofonoff, 1962) are related to the definition of GPE introduced in the present study. The dynamic height $\Delta D$ is the potential energy per unit mass relative to a reference ocean with temperature $0^{\circ} \mathrm{C}$ and salinity $35 \%$. The anomaly of potential energy $\chi$ is defined as the potential energy per unit area found by integrating $\Delta D$ over the water column from surface to pressure $p$. The classical reference field is not attainable by isentropic processes from the initial field and, therefore, has no dynamical or thermodynamical interpretation.

The isentropically leveled field defines a set of material surfaces within the ocean with respect to adiabatic displacement. The corresponding surfaces for the initial field have the same distribution of salinity, potential temperature and presumably other conservative tracers as the reference field that are 
unchanged by an isentropic displacement relative to the pressure field. Hence, they form the appropriate surfaces for identifying water masses by tracer properties. Montgomery (1938) approximated these surfaces by potential density $\left(\sigma_{\theta}\right)$ or sigma- $t\left(\sigma_{t}\right)$ (densities referred adiabatically or isothermally to atmospheric pressure). As the vertical displacements are usually small compared to the depth, use of surface pressure as a reference is not obviously as appropriate for local processes as a reference surface at the mean pressure. Lynn and Reid (1968), for example, introduced density referred to $4000 \mathrm{db}$ as a closer approximation to a tracer surface in their study of deep-water circulation. Because of the strong dependence of the thermal expansion coefficient on pressure, $\sigma_{\theta}$ cannot be used to label material surfaces in deep water. The procedure used in the present study is equivalent to introducing a separate reference surface at each pressure.

Because of the dependence of density on both temperature and salinity, the material surfaces cannot be characterized by a single explicit variable such as potential temperature or $\sigma_{\theta}$. The appropriate single parameter is the density or specific volume of the reference field. This variable has to be calculated from the three-dimensional initial density field for the region selected. It is not explicitly known beforehand. The implicit character of the reference field has been an obstacle to study of available potential energy both in theoretical studies and in interpretation of data. The present study provides a basis for a more accurate estimation of available potential energy from measurements of the density field and an assessment of the errors involved in using simpler perturbation methods.

\section{Potential energy}

Because vertical position cannot be measured accurately, the potential energy of a column of seawater cannot be determined absolutely. Estimates are made relative to an unknown local reference level that is not transferable from one measurement point to another. It is more convenient, therefore, to introduce a pressure surface as a reference because pressure is measured directly. The distribution of geopotential on the reference pressure surface is unknown and cannot be determined from the density field alone. Thus the estimates of potential energy are undetermined to the extent of an unknown function of horizontal position corresponding to the unknown geopotential on the selected reference pressure surface. An equivalent interpretation is that the pressure field relative to geopotential surfaces is unknown to the extent of an arbitrary barotropic pressure field that is a function of horizontal position only.

The total potential energy $E_{T}$ of a column of seawater of unit horizontal cross section, relative to a reference level $z_{r}$, is given by

$$
E_{T}=\int_{z_{r}}^{z_{s}} \rho\left(\phi-\phi_{r}\right) d z,
$$

where $\rho$ is density (in situ), $\phi$ geopotential and $z_{s}$ the surface of the ocean measured along the vertical coordinate $z$. The integral is evaluated in terms of pressure, assuming hydrostatic balance, by substituting $d p=-\rho g d z=-\rho d \phi$, where $g=d \phi / d z$ is gravity, to obtain

$$
E_{T}=\int_{p_{s}}^{p_{r}}\left(\phi-\phi_{r}\right) / g d p,
$$

where $p_{s}$ and $p_{r}$ are pressures at the surface $z_{s}$ and the reference level $z_{r}$, respectively. For convenience, the oceanographic convention $p_{s}=0$ is used subsequently.

From the hydrostatic equation, the geopotential difference $\phi-\phi_{r}$ is

$$
\phi-\phi_{r}=\int_{p}^{p_{r}} \alpha d p^{\prime},
$$

where $\alpha=1 / \rho$ is specific volume, so that

$$
E_{T}=\int_{0}^{p_{r}} g^{-1} \int_{p}^{p_{r}} \alpha d p^{\prime} d p,
$$

expressing the total potential energy relative to $p_{r}$ in terms of the specific volume measured as a function of pressure and horizontal position.

Assuming that the reference field of specific volume $\alpha_{f}$ is known as a function of pressure only (the procedure for estimating $\alpha_{f}$ is outlined in Section 6), the total potential energy relative to the adiabatic minimum is

$E_{T}^{i}-E_{T}^{f}=\int_{0}^{p_{r}} g^{-1} \int_{p}^{p_{r}}\left(\alpha_{i}-\alpha_{f}\right) d p^{\prime} d p \equiv E\left(0, p_{r}\right)$,

where $\alpha_{i}$ is the observed field and $\alpha_{f}$ the adiabatically leveled field for the region considered.

Contributions to the total potential energy can be examined within the water column by introducing the potential energy function

$$
E\left(p, p_{r}\right)=\int_{p}^{p_{r}} g^{-1} \int_{p^{\prime}}^{p_{r}}\left(\alpha_{i}-\alpha_{f}\right) d p^{\prime \prime} d p^{\prime}
$$

integrated from a pressure $p$ to the reference pressure $p_{r}$.

The potential energy function $E\left(p, p_{r}\right)$ can be expressed in terms of the anomaly of dynamic height $\Delta D$ and anomaly of potential energy $\chi$ (Fofonoff, 1962) redefined as

$$
\Delta D=\int_{0}^{p}\left(\alpha_{i}-\alpha_{f}\right) d p^{\prime},
$$




$$
\chi=\int_{0}^{p} \frac{p}{g}\left(\alpha_{i}-\alpha_{f}\right) d p^{\prime} .
$$

These differ from the classical definitions in the choice of the reference specific volume field, i.e., $\alpha_{f}(p)$ rather than $\alpha_{35,0, p}$.

The anomaly of potential energy $\chi$ yields potential energy of a column of water between the surface $(p=0)$ and $p$, measured relative to the pressure surface $p$. The pressure surface at $p$ is displaced vertically by $\Delta z=\left[\Delta D\left(p_{r}\right)-\Delta D(p)\right] / g$ relative to the reference surface $p_{r}$. Hence, the total potential energy from 0 to $p$ referenced to $p_{r}$ is given by

$$
\begin{aligned}
E\left(0, p_{r}\right)-E\left(p, p_{r}\right) & =\chi(p)+p g^{-1}\left[\Delta D\left(p_{r}\right)-\Delta D(p)\right] \\
& =\int_{0}^{p} g^{-1}\left[\Delta D\left(p_{r}\right)-\Delta D(p)\right] d p^{\prime}, \\
& =\int_{0}^{p} g^{-1} \int_{p^{\prime}}^{p_{r}}\left(\alpha_{i}-\alpha_{f}\right) d p^{\prime \prime} d p^{\prime} . \quad \text { (9) }
\end{aligned}
$$

Similarly, the potential energy between two pressure surfaces $p_{1}$ and $p_{2}$ is

$$
\begin{gathered}
E\left(p_{1}, p_{r}\right)-E\left(p_{2}, p_{r}\right)=\int_{p_{1}}^{p_{2}} g^{-1}\left[\Delta D\left(p_{r}\right)-\Delta D(p)\right] d p \\
=\chi\left(p_{2}\right)-\chi\left(p_{1}\right)+p_{2} g^{-1}\left[\Delta D\left(p_{r}\right)-\Delta D\left(p_{2}\right)\right] \\
-p_{1} g^{-1}\left[\Delta D\left(p_{r}\right)-\Delta D\left(p_{1}\right)\right] . \quad \text { (10) }
\end{gathered}
$$

The contribution per unit mass is

$g \frac{d E}{d p}=\left[\Delta D\left(p_{r}\right)-\Delta D(p)\right]=\int_{p}^{p_{r}}\left(\alpha_{i}-\alpha_{f}\right) d p^{\prime}$.

Horizontal gradients of the potential energy function $E\left(p, p_{r}\right)$ are related to geostrophic mass transport. If $E_{1}$ and $E_{2}$ are the potential energies at two horizontally separated points, $x_{1}, x_{2}$, the transport $T_{r}$ through the section joining the two points, relative to $p_{r}$, is given by

$$
T_{r}=\left(E_{2}-E_{1}\right) / f,
$$

where $f$ is the Coriolis parameter. The result can be obtained by direct integration of the geostrophic equation

$$
\begin{aligned}
T_{r} & =\int_{x_{1}}^{x_{2}} \int_{z}^{z_{s}} \rho\left(v-v_{r}\right) d z^{\prime} d x \\
& =\int_{x_{1}}^{x_{2}} \int_{0}^{p} \frac{1}{g f} \frac{\partial}{\partial x}\left(\Delta D_{r}-\Delta D\right) d p^{\prime} d x \\
& =\left.f^{-1} E\left(p, p_{r}\right)\right|_{x_{1}} ^{x_{2}}=f^{-1}\left(E_{2}-E_{1}\right) .
\end{aligned}
$$

\section{Available gravitational potential energy (GPE)}

The potential energy $E$ defined previously can be positive or negative depending on whether the isosteric surfaces $\alpha_{i}$ are displaced to higher or lower pressures relative to $\alpha_{f}$. However, there is a net positive storage of potential energy for a stably stratified fluid, i.e., $\bar{E}>0$, where $\bar{E}$ is averaged horizontally over the area under consideration. This net positive storage of potential energy per unit area is identified as the total available gravitational potential energy (TGPE)

$$
\text { TGPE }=A^{-1} \iint E\left(0, p_{r}\right) d x d y=\bar{E},
$$

where $A$ is the area of horizontal averaging.

Contributions to the total available gravitational energy are defined as

$$
\begin{aligned}
\operatorname{TGPE}(p) & =\bar{E}(p) \\
& =\int_{p}^{p_{r}} g^{-1} \int_{p^{\prime}}^{p_{r}}\left(\bar{\alpha}_{i}-\alpha_{f}\right) d p^{\prime \prime} d p^{\prime},
\end{aligned}
$$

with units of potential energy per unit area $\left(\mathrm{J} \mathrm{m}^{-2}\right)$ and

$$
\begin{aligned}
\operatorname{GPE}(p) & =g \frac{d \mathrm{TGPE}}{d p} \\
& =\int_{p}^{p_{r}}\left(\bar{\alpha}_{i}-\alpha_{f}\right) d p^{\prime}=\left(\overline{\Delta D_{r}-\Delta D}\right),
\end{aligned}
$$

with units of potential energy per unit mass $\left(\mathrm{J} \mathrm{kg}^{-1}\right)$.

The definition given in (14) for GPE (per unit mass) is useful for examining the vertical distribution of contributions to GPE. Its relationship to the net available potential energy is examined in the next two sections.

\section{Work by pressure gradients}

For a stably stratified fluid in a gravity field, external work against pressure-gradient forces is required to displace density surfaces relative to geopotential surfaces. If the displacements are adiabatic and reversible and sufficiently slow that kinetic energy is negligible in the displacement, the work done must equal the work regained in relaxing the field back to its initial configuration. We can identify the work required to produce the displacements as the net or "true" available potential energy.

If the fluid is incompressible, the displacements can redistribute internal energy within the field but cannot change the total internal energy. Each fluid element preserves its volume and, therefore, its internal energy. The work must appear as a change of gravitational potential energy only, i.e., there must be a net shift of mass relative to geopotential surfaces and a corresponding change of mean density and pressure along fixed geopotential surfaces. If the fluid is compressible, the change of density or, equivalently, the change of specific volume and pressure will alter the internal energy. Thus, some of the work 


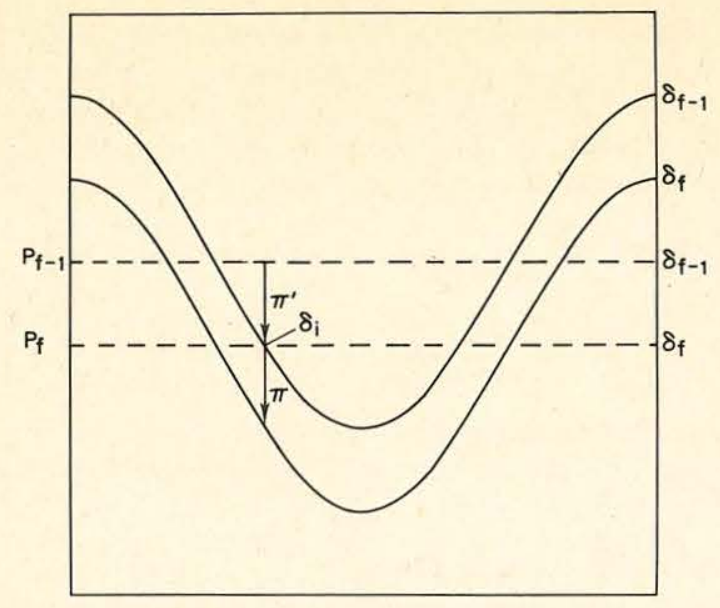

FIG. 1. The initial, observed steric anomaly field (solid lines) is assumed to be formed by vertical displacement of a reference steric anomaly field $\delta_{f}$ (dashed lines) that is uniform on pressure surfaces. The observed steric anomaly $\delta_{i}$ on pressure surface $p_{f}$ is the reference surface $\delta_{f-1}$ displaced adiabatically by $\pi^{\prime}$ from the pressure surface $p_{f-1}$.

done on the fluid will appear as internal energy. If internal energy is increased by the displacements as is usually the case in the atmosphere, the total potential energy change is less than the work done to produce the displacement. In the ocean, the internal energy change is usually negative, so that the total potential energy change is greater than the work done.

For the ocean, the internal energy contributions are small (10-20\% of GPE). Hence, estimates of APE can be made by computing GPE from (14) and adding the internal energy contribution separately. This procedure provides an alternative estimate of APE that is not restricted to small amplitudes inherent in the Boussinesq approximation. Estimates of both GPE and internal energy contributions can be obtained in terms of a Taylor series expansion about the reference field. These are examined in Section 5.

\section{Perturbation of the reference field}

The relationship between the observed and leveled reference fields can be developed as a Taylor series expansion about the reference field assuming adiabatic displacements. The expansion shows the connection with the linear Boussinesq approximation and with the internal energy and enthalpy changes within the density field.

The observed (initial) field of specific volume $\alpha_{i}$ (or any other thermodynamic function of the independent variables pressure $p$, temperature $\theta$ and salinity $S$ ) is assumed to be displaced adiabatically from its reference pressure $p$ by a pressure interval $\pi$, which is a function of position, pressure and time as illustrated in Fig. 1. The initial and final fields are linked by reversible adiabatic processes defined by

where

$$
\alpha_{i}(p)=\alpha_{f}\left(p-\pi^{\prime}\right)+\int_{p-\pi^{\prime}}^{p}\left(\frac{\partial \alpha}{\partial p}\right)_{a} d p^{\prime},
$$

$$
\pi^{\prime}=\pi\left(p-\pi^{\prime}\right) \text {. }
$$

The observed specific volume $\alpha_{i}$ at pressure $p$ is assumed to be the reference specific volume $\alpha_{f}$ displaced adiabatically from $p-\pi^{\prime}$ to $p$ (Fig. 1). Because the displacements $\pi$ vary with depth, $\pi^{\prime}$ and $\pi$ differ. The reference specific volume corresponding to pressure $p$ is located at $p+\pi$ in the observed field. Horizontal displacements are neglected compared with the horizontal scale of the density field. Expanding (15) about $p$ to second order in $\pi^{\prime}$ yields

$$
\begin{aligned}
& \alpha_{i}(p)=\alpha_{f}(p)-\frac{d \alpha_{f}}{d p} \pi^{\prime}+\frac{1}{2} \frac{d^{2} \alpha_{f}}{d p^{2}} \pi^{\prime 2} \\
& +\kappa \pi^{\prime}-\frac{d \kappa}{d p} \pi^{\prime 2}+\frac{1}{2}\left(\frac{\partial \kappa}{\partial p}\right)_{a} \pi^{\prime 2}+\mathrm{O}\left(\pi^{\prime 3}\right), \\
& \pi^{\prime}=\pi-\frac{d \pi}{d p} \pi^{\prime}+\cdots=\pi-\frac{d^{1} / 2 \pi^{2}}{d p}+\mathrm{O}\left(\pi^{\prime 3}\right),
\end{aligned}
$$

where $\kappa=(\partial \alpha / \partial p)_{a}$, is the adiabatic derivative with respect to pressure.

The change of specific volume at $p$ resulting from adiabatic leveling is given by

$$
\begin{array}{r}
\Delta \alpha=\alpha_{i}(p)-\alpha_{f}(p)=-\alpha_{p}^{*} \pi^{\prime}+\frac{1}{2}\left(\frac{d}{d p} \alpha_{p}^{*}\right) \pi^{\prime 2} \\
-1 / 2 \Gamma_{\kappa} \pi^{\prime 2}+\mathrm{O}\left(\pi^{\prime 3}\right),
\end{array}
$$

where

$$
\begin{aligned}
& \alpha_{p}^{*}=\frac{d \alpha}{d p}-\kappa, \\
& \Gamma_{\kappa}=\frac{d \kappa}{d p}-\left(\frac{\partial \kappa}{\partial p}\right)_{a} .
\end{aligned}
$$

Substituting for $\pi^{\prime}$ in (18) gives

$\Delta \alpha=-\alpha_{p}^{*} \pi+\frac{d}{d p}\left(1 / 2 \alpha_{p}^{*} \pi^{2}\right)-1 / 2 \Gamma_{\kappa} \pi^{2}+\mathrm{O}\left(\pi^{3}\right)$

to second order in $\pi$. More generally, the change of any thermodynamic function $\phi$ is given by

$\Delta \phi=-\phi_{p}^{*} \pi+\frac{d}{d p}\left(1 / 2 \phi_{p}^{*} \pi^{2}\right)$

$$
-1 / 2 \Gamma_{\phi} \pi^{2}+\mathrm{O}\left(\pi^{3}\right)
$$

where

$$
\begin{aligned}
& \phi_{p}^{*}=\frac{d \phi}{d p}-\left(\frac{\partial \phi}{\partial p}\right)_{a}, \\
& \Gamma_{\phi}=\frac{d}{d p}\left(\frac{\partial \phi}{\partial p}\right)_{a}-\left(\frac{\partial^{2} \phi}{\partial p^{2}}\right)_{a} .
\end{aligned}
$$


Thus, internal energy changes $\Delta e$ associated with the leveling process are given by

$\Delta e=\Delta h_{q}+p \alpha_{p}^{*} \pi-\frac{d}{d p}\left(1 / 2 p \alpha_{p}^{*} \pi^{2}\right)+1 / 2 p \Gamma_{\kappa} \pi^{2}$

and enthalpy changes $\Delta h$ by

where

$$
\Delta h=\Delta h_{q}-1 / 2 \alpha_{p}^{*} \pi^{2},
$$

$$
\begin{aligned}
\Delta h_{q} & =-h_{q p} \pi+\frac{d}{d p}\left(1 / 2 h_{q p} \pi^{2}\right), \\
h_{q p} & =T \frac{d \eta}{d p}+\mu \frac{d S}{d p},
\end{aligned}
$$

and $T$ is absolute temperature, $S$ salinity, $\eta$ specific entropy and $\mu$ relative chemical potential (Fofonoff, 1962).

Similarily, changes of the conserved variables entropy and salinity on the pressure surface $p$ are given by

$$
\begin{aligned}
& \Delta S=-\frac{d S}{d p} \pi+\frac{d}{d p}\left(\frac{1}{2} \frac{d S}{d p} \pi^{2}\right), \\
& \Delta \eta=-\frac{d \eta}{d p} \pi+\frac{d}{d p}\left(\frac{1}{2} \frac{d \eta}{d p} \pi^{2}\right) .
\end{aligned}
$$

As the salinity and entropy are unchanged for every fluid element undergoing adiabatic displacements, integrals of $\Delta S, \Delta \eta$ and $\Delta_{h q}$ must be zero for the area considered. The changes represent redistribution of the conserved variables with no internal sources or sinks.

The difference of total salt content $\Delta S_{T}$ per unit area between two constant salinity surfaces $S_{1}, S_{2}$ corresponding to pressures $p_{1} p_{2}$, is given by

$$
\begin{aligned}
g \Delta S_{T} & =\int_{p_{1}+\pi_{1}}^{p_{2}+\pi_{2}} S^{i} d p-\int_{p_{1}}^{p_{2}} S^{f} d p \\
& =-\int_{S_{1}}^{S_{2}} \pi d S+\pi_{2} S_{2}-\pi_{1} S_{1} .
\end{aligned}
$$

The horizontal average $\overline{\Delta S_{T}}$ is zero if it is assumed that the field is in hydrostatic equilibrium and no mass exchange occurs across salinity surfaces for the adiabatic displacements. Along each such surface $\bar{\pi}=0$. Hence, total salt content is unchanged.

The integral in (24) can be written

$$
g \Delta S_{T}=\int_{p_{1}}^{p_{2}} \Delta S d p+\int_{p_{2}}^{p_{2}+\pi_{1}} S^{i} d p-\int_{p_{1}}^{p_{1}+\pi_{1}} S^{i} d p .
$$

Substitution of the Taylor expansion from (23) and expanding $S^{i}$ about $p_{1}$ and $p_{2}$ yields

$$
\begin{aligned}
g \Delta S_{T}=-\int_{p_{1}}^{p_{2}} \frac{d S}{d p} \pi d p & +\left[S^{i}\left(p_{2}\right)+\frac{d S}{d p} \pi_{2}\right] \pi_{2} \\
& -\left[S^{i}\left(p_{1}\right)+\frac{d S}{d p} \pi_{1}\right] \pi_{1},
\end{aligned}
$$

which is equivalent to (24) to $\mathrm{O}\left(\pi^{3}\right)$. Similar arguments hold for other conserved variables.

The dynamic height relative to a reference pressure $p_{r}$ is found by integrating (18) with respect to pressure, i.e.,

$$
\begin{aligned}
\Delta D\left(p_{r}\right) & -\Delta D(p)=\int_{p}^{p_{r}} \Delta \alpha d p^{\prime}=-\int_{p}^{p_{r}} \alpha_{p}^{*} \pi d p^{\prime} \\
& +1 / 2 \alpha_{p}^{*} \pi_{r}^{2}-1 / 2 \alpha_{p}^{*} \pi^{2}-\frac{1}{2} \int_{p}^{p_{r}} \Gamma_{\kappa} \pi^{2} d p^{\prime} .
\end{aligned}
$$

The term $-1 / 2 \alpha_{p}^{*} \pi_{r}^{2}$ is the contribution to the height anomaly at the reference pressure $p_{r}$. This term can be evaluated from measurements and used as a boundary condition at $p_{r}$ so that the height anomaly $\Delta Z$ is

$$
\begin{aligned}
& g \Delta Z=\Delta D\left(p_{r}\right)-\Delta D(p)-1 / 2 \alpha_{p}^{*} \pi_{r}{ }^{2} \\
& =-\int_{p}^{p_{r}} \alpha_{p}^{*} \pi d p^{\prime}-1 / 2 \alpha_{p}^{*} \pi^{2}-\frac{1}{2} \int_{p}^{p_{r}} \Gamma_{\kappa} \pi^{2} d p^{\prime} .
\end{aligned}
$$

Hence, GPE corresponding to (14) is

$$
\begin{aligned}
\mathrm{GPE} & =g \frac{d \mathrm{TGPE}(p)}{d p} \\
& =-\int_{p}^{p_{r}} \overline{\alpha_{p}^{*} \pi} d p^{\prime}-1 / 2 \overline{\alpha_{p}^{*} \pi^{2}}-\frac{1}{2} \int_{p}^{p_{r}} \overline{\Gamma_{\kappa} \pi^{2}} d p^{\prime} .
\end{aligned}
$$

The specific volume surfaces $\alpha_{f}$ remain material surfaces during the adiabatic displacement. There is no mass exchange across the surface so that $\bar{\pi}=0$ averaged over the area considered. As $\alpha_{f}$ is constant along each pressure surface its derivative $d \alpha_{f} /$ $d p$ must also be constant. Therefore,

$$
-\overline{\alpha_{p}^{*} \pi}=-\overline{\left(\frac{d \alpha_{f}}{d p}-\kappa\right) \pi}=\overline{\kappa \pi} .
$$

If the reference surface has variations of temperature as a function of horizontal position, the adiabatic compressibility will not be uniform and $\overline{\kappa \pi}$ $\neq 0$. This is interpreted to mean that the average specific volume of the surface $\alpha_{f}$ changes during the relaxation, yielding corresponding changes in the average dynamic height and GPE. These contributions arise from horizontal inhomogeneities of $\kappa$, and represent a conversion between internal and potential energy.

The second term in (28) is familiar from linear Boussinesq theory. It can be expressed in the form

$$
-1 / 2\left(\overline{\alpha_{p}^{*} \pi^{2}}\right)=1 / 2\left(\overline{N^{2} \xi^{2}}\right),
$$

where $N$ is the local buoyancy frequency and $\xi$ $=-\pi / \rho g$ the local vertical displacement corresponding to the pressure perturbation $\pi$. The last term in (28) arises from volume changes associated with the pressure changes on each fluid element. If the temperature decreases with pressure, leveling results in 
colder water moving to higher pressure and warmer water to lower pressure. Because colder water is more compressible, there is a net reduction of volume associated with the transition to the reference state yielding a lower reference potential energy. Potential energy is converted to internal energy by this volume decrease. The contribution can be of either sign depending on the vertical gradients of temperature and salinity. These contributions arise from vertical inhomogeneities of $\kappa$.

\section{Computational procedure for determining the ref- erence field}

The distribution of temperature $T$ and salinity $S$ is measured as a function of pressure by lowering sensors through the water column from shipboard. Salinity is usually computed indirectly from electrical conductivity (Fofonoff et al., 1974).

It can be assumed that a set of hydrographic stations irregularly spaced in horizontal position and time are available within some region of the ocean. Questions of adequacy of sampling and instrumental accuracy are deferred to Section 9.

For each station, a series of pressure levels is selected for computation of the reference density field. The location and number of pressure levels is determined by the vertical resolution required in the particular region considered. Uniform spacing of pressure intervals is not required.

For convenience, the computations are carried out in terms of specific volume anomaly (steric anomaly) defined as

$$
\delta=\alpha(p, T, S)-\alpha(p, 0,35)
$$

and computed from observed values of pressure, temperature and salinity. The Knudsen-Ekman equation of state is used throughout (Fofonoff, 1962).

The potential steric anomaly $\delta_{f}$ of the reference field corresponding to pressure $p_{f}$ is found by fitting least-squares-regression polynomials of pressure and potential temperature in terms of potential steric anomaly within a local pressure interval $\Delta p$ about $p_{f}$ for each station at each pressure level. The interval $\Delta p$ must be chosen larger than the maximum displacement of the reference surface, i.e., $\mid p_{i}$ $-\left.p_{f}\right|_{\max }<\Delta p$, where $p_{i}$ is the observed pressure of the deformed reference field. The intervals can be overlapped if higher resolution is desired.

Within each interval $\Delta p$, the potential temperature $\theta$ (Fofonoff, 1977) is given by

$$
\theta\left(p, p_{f}\right)=T(p)+\int_{p}^{p_{f}}\left(\frac{\partial T}{\partial p}\right)_{a} d p^{\prime}
$$

and the potential steric anomaly by

$$
\delta\left(p, p_{f}\right)=\delta\left[p_{f}, \theta\left(p, p_{f}\right), S(p)\right],
$$

i.e., all steric anomalies are referred adiabatically to $p_{f}$.

The regression polynomials for station $k$ are

$$
\begin{aligned}
& \hat{p}_{k}=\sum_{n=0}^{N} \alpha_{k n}\left(\delta-\delta_{k}\right)^{n}, \\
& \hat{\theta}_{k}=\sum_{n=0}^{N} \beta_{k n}\left(\delta-\delta_{k}\right)^{n},
\end{aligned}
$$

where $\delta_{k}$ is the average over $\Delta p$. Standard regression methods are used to determine $\alpha_{k n}, \beta_{k n}$ (Brownlee, 1965). The degree $N$ of the polynomial and the pressure interval $\Delta p$ as well as the separation of pressure levels can be varied with depth to maintain optimum fit and vertical resolution. Optimization is discussed in Section 9.

In addition, an estimate is made of the variance $\sigma^{2}$ of a single data point, either pressure or temperature, from the regression residuals (Fofonoff and Bryden, 1975):

$$
\begin{aligned}
& \sigma_{p}{ }^{2}=\frac{1}{Q-N} \sum_{\nu=1}^{Q}\left(p_{\nu}-\hat{p}_{\nu}\right)^{2}, \\
& \sigma_{\theta}{ }^{2}=\frac{1}{Q-N} \sum_{\nu=1}^{Q}\left(\theta_{\nu}-\hat{\theta}_{\nu}\right)^{2},
\end{aligned}
$$

with $Q$ the number of observations in $\Delta p, p_{\nu}, \theta_{v}$ the observed pressure and potential temperature, and $\hat{p}_{\nu}, \hat{\theta}_{v}$ the regression estimates of $p$ and $\theta$ at $\delta_{v}$. Further, estimates of the variances of the regression estimates $\hat{p}, \hat{\theta}$ at any value of $\delta$ are given by

$$
\begin{aligned}
V(\hat{p}(\delta)) & =\sigma_{p}{ }^{2} \sum_{i=1}^{N+1} \sum_{j=1}^{N+1} R_{i j}^{-1}\left(\delta-\delta_{k}\right)^{i+j-2}, \\
V(\hat{\theta}(\delta)) & =\sigma_{\theta}^{2} \sum_{i=1}^{N+1} \sum_{j=1}^{N+1} R_{i j}^{-1}\left(\delta-\delta_{k}\right)^{i+j-2}, \\
R_{i j} & =\sum_{\nu=1}^{Q}\left(\delta_{\nu}-\delta_{k}\right)^{i+j-2} .
\end{aligned}
$$

These variances will be used in the error analysis in Section 9.

The regression polynomials provide smoothed local relationships between pressure, potential temperature and potential steric anomaly. Salinity is implicitly determined by these polynomials. For a given value of $\delta_{f}$, these polynomials specify the pressure of the steric anomaly surface referred adiabatically to $p_{f}$, and the potential temperature on the surface. Additional regression polynomials can be fitted to other tracers as required.

No mass exchange can occur across the surface $\delta_{f}$ in moving adiabatically from the observed field to the reference field. As the mass above the surface is $p_{i} / g$ initially and $p_{f} / g$ in the reference field, this constraint is met by specifying 


$$
\overline{p_{k}\left(\delta_{f}\right)}=p_{f}=\sum_{k=1}^{M} \omega_{k} \hat{p}_{k}\left(\delta_{f}\right),
$$

i.e., that the horizontal average of pressure over the initial steric surface corresponding to $\delta_{f}$ be unchanged by the deformation. Weights $\omega_{k}$ are assigned to each of the $M$ stations in the data set. These are defined in Section 7.

The pressure polynomials are shifted to a common origin $\delta_{m}$ for averaging:

$\hat{p}_{k}=\sum_{n=0}^{N} \alpha_{k n}\left(\delta-\delta_{m}+\Delta_{k}\right)^{n}=\sum_{n=0}^{N} \alpha_{k n}^{\prime}\left(\delta-\delta_{m}\right)^{n}$,

where

$$
\begin{aligned}
\Delta_{k} & =\delta_{m}-\delta_{k}, \\
\delta_{m} & =\overline{\delta_{k}} \text { averaged over } M \text { stations, } \\
\alpha_{k n}^{\prime} & =\sum_{r=0}^{N-n} \alpha_{k, n+r} \frac{(n+r) !}{n ! r !} \Delta_{k}^{r} .
\end{aligned}
$$

Averaging $\hat{p}_{k}\left(\delta_{f}\right)$ over the region with weights $\omega_{k}$ yields a single polynomial

$p_{f}=\sum_{n=0}^{N} \sum_{k=1}^{M} \omega_{k} \alpha_{k n}^{\prime}\left(\delta_{f}-\delta_{m}\right)^{n}=\sum_{n=0}^{N} \overline{\alpha_{n}}\left(\delta_{f}-\delta_{m}\right)^{n}$,

which is inverted by standard iterative methods to determine $\delta_{f}$.

Given $\delta_{f}$, the displacement $\pi_{k}=\hat{p}_{k}\left(\delta_{f}\right)-p_{f}$ of the surface $\delta_{f}$ from $p_{f}$ in the observed field and the potential temperature on the surface are found by substitution into (30) and (31). The smoothed initial steric anomaly $\delta_{i k}$ on $p_{f}$ is obtained by inverting

$$
p_{f}=\sum_{n=0}^{N} \alpha_{k n}\left(\delta_{i k}-\delta_{k}\right)^{n} \text {. }
$$

Care must be taken to exclude spurious roots that fall outside of the range of the regression polynomial. The procedure uses the general equation of state and can be made as precise as desired within the limitations of accuracy of the data.

The dynamic height and anomaly of potential energy are obtained by numerical integration of

$$
\begin{aligned}
\Delta D_{k} & =\int_{0}^{p}\left(\delta_{i k}-\delta_{f}\right) d p^{\prime}, \\
\chi_{k} & =g^{-1} \int_{0}^{p} p\left(\delta_{i k}-\delta_{f}\right) d p^{\prime},
\end{aligned}
$$

over the series of pressure levels at each station. The contribution to GPE at pressure $p$, for example, is given by

$$
\begin{aligned}
\mathrm{GPE} & \left.=\int_{p}^{p_{r}} \overline{\left(\delta_{i k}\right.}-\delta_{f}\right) d p^{\prime} \\
& =\sum_{k=1}^{M} \omega_{k} g^{-1}\left[\Delta D_{k}\left(p_{r}\right)-\Delta D_{k}(p)\right] .
\end{aligned}
$$

\section{Averaging weights $\omega_{k}$}

The weights $\omega_{k}$ assigned to each station were determined by objective mapping techniques (Gandin, 1965; Bretherton et al., 1976). Given a scalar $p_{k}$ at $x_{k}, y_{k}$, the interpolated field over $A$ is

$$
p(x, y)=\sum_{k=1}^{M} C\left(x, y, x_{k}, y_{k}\right) \eta_{k},
$$

where $C$ is the correlation function for $p$ in $A$. For simplicity, the correlation function is assumed to be homogeneous and isotropic in $A$, dependent only on the separation between $x, y$ and $x_{k} y_{k}$. The coefficients are obtained by solving the set of linear equations

where

$$
p\left(x_{i}, y_{i}\right)=\sum_{k=1}^{M} C_{i k} \eta_{k},
$$

$$
C_{i k}=C\left(x_{i}, y_{i}, x_{k}, y_{k}\right)+\epsilon \delta_{i k} ; \quad \delta_{i k}= \begin{cases}0, & i \neq k \\ 1, & i=k\end{cases}
$$

is a symmetric correlation matrix and $\epsilon$ (assumed constant over $A$ ) the contribution of uncorrelated noise at each point. Thus,

$$
\eta_{k}=\sum_{i=1}^{M} C_{k i}^{-1} p\left(x_{i}, y_{i}\right)
$$

where $C_{k i}^{-1}$ is the inverse matrix of $C_{k i}$.

The integral of $C\left(x, y, x_{k}, y_{k}\right)$ over $A$ under present assumptions is a constant, $C_{0}$, independent of $x_{k}, y_{k}$. Therefore,

$$
\begin{aligned}
\bar{p} & =A^{-1} \int p(x, y) d x d y=C_{0} A^{-1} \sum_{k=1}^{M} \eta_{k} \\
& =C_{0} A^{-1} \sum_{k=1}^{M} \sum_{i=1}^{M} C_{k i}^{-1} p\left(x_{i}, y_{i}\right)=\sum_{i=1}^{M} \omega_{i} p\left(x_{i}, y_{i}\right) .
\end{aligned}
$$

The weights are

$$
\omega_{i}=\sum_{j=1}^{M} C_{i j}^{-1} / \sum_{i=1}^{M} \sum_{j=1}^{M} C_{i j}^{-1},
$$

normalized so that

$$
\sum_{i=1}^{M} \omega_{i}=1 .
$$

Other schemes for weighting each station are possible. The procedure given above provides an explicit weighting for an irregular station grid.

\section{MODE computations}

Five groups of density stations (designated Groups A-E) from the MODE field experiment were selected for analysis. These were chosen during periods of maximum spatial coverage. The stations used in each 
TABLE 1 . MODE density data selected for analysis.

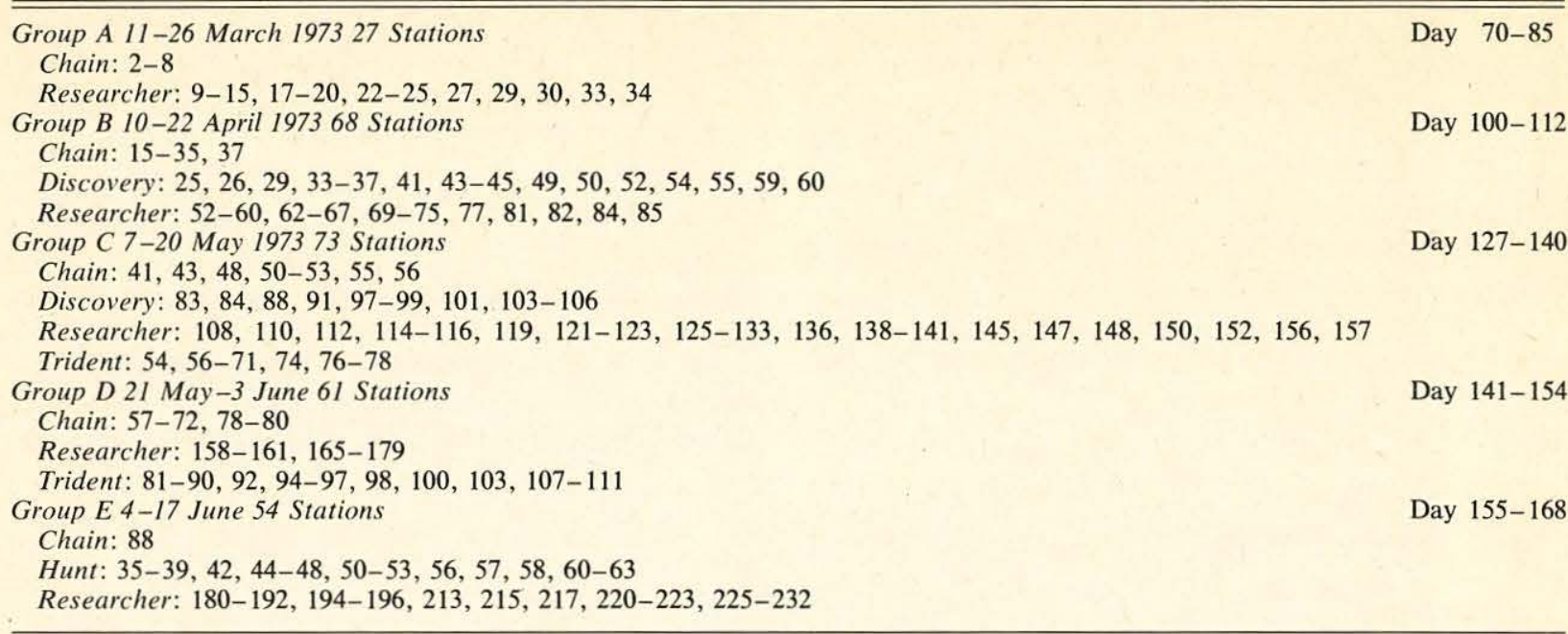

Group are listed in Table 1. Selection criteria were as follows:

- good salinities

- data to $2800 \mathrm{db}$

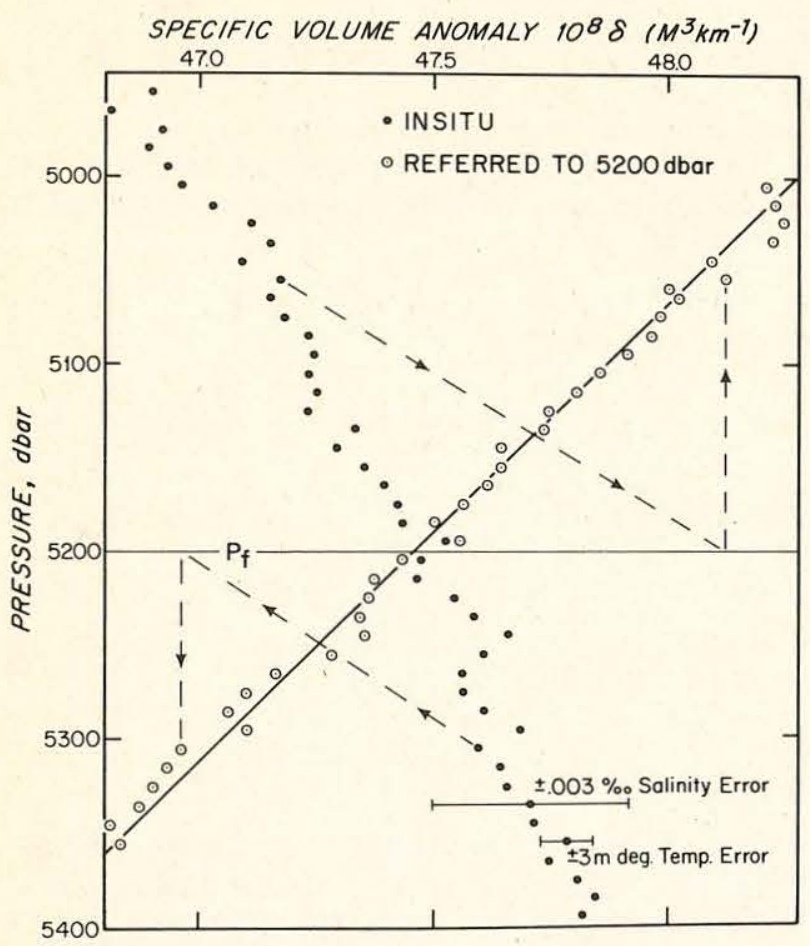

FIG. 2. A sample regression for deep water showing in situ steric anomalies (solid dots) in the regression interval. Each observation is referenced adiabatically to the reference pressure $p_{f}$ and replotted at its original pressure (open dots). The regression curve is fitted to the open dots. Effects of measurement errors of temperature and salinity are indicated for single observations. Each solid dot represents a $10 \mathrm{db}$ average containing $\sim 50-200$ separate measurements depending on the instrument used. Most of the scatter is attributed to finestructure within the layer.
- no data gaps exceeding $20 \mathrm{db}$ in the upper $2800 \mathrm{db}$ - $>10 \mathrm{~km}$ separation from neighboring stations.

All stations selected consisted of $2 \mathrm{db}$ interval averages of original data. These were passed through an editing program that fitted a cubic least-squaresregression polynomial to the temperature and salinity values for consecutive groups of 16 data cycles each. Values exceeding three standard deviations from the regression polynomial were replaced by an interpolated value obtained from the regression-polynomial fitted after rejection of the data point. The selected data scans were then averaged arithmetically in non-overlapping groups of five cycles each to form $10 \mathrm{db}$ interval stations. A total of 283 stations were selected for the five groups.

For each station, regression coefficients and standard deviations of residuals for pressure and potential temperature referenced to $p_{f}$ were calculated. An example of the fitting procedure is given in Fig. 2 for a deep-water level. The reference steric anomaly $\delta_{f}$ at each pressure was computed by averaging the regression coefficients for all stations in each group and inverting the resultant averaged polynomial for $\delta_{f}$. The vertical displacements $\pi$ and potential temperature $\theta_{f}$ were calculated from the regression polynomials for each station. Two sets of regression parameters $\left(N, \Delta p, p_{f}\right)$ as functions of depth for the MODE data are summarized in Table 2. The highresolution case represents the optimized calculation; the low-resolution case is provided for comparison.

The reference steric anomaly field $\delta_{f}$ changed significantly from one group to the next, indicating shifts of the depths of the reference surface over the station grid of as much as $30 \mathrm{db}$. The potential energy changes associated with the changes of the reference field are much larger than the GPE of the density structure within the station grid for each group 
TABLE 2. Pressure levels $p_{f}$, regression interval $\Delta p=p_{\max }-p_{\min }$, and polynomial order $N$ for two cases selected for MODE data: high vertical resolution and low vertical resolution (e.g., in the high-resolution case: from $p_{f}=50$ to $p_{f}=200$ the integration step (in parentheses) is $50 \mathrm{db}, \Delta p$ is $200 \mathrm{db}$, and $N$ is 6).

\begin{tabular}{|c|c|c|c|c|c|c|c|c|c|}
\hline \multicolumn{5}{|c|}{ High vertical resolution } & \multicolumn{5}{|c|}{ Low vertical resolution } \\
\hline & $p_{f}$ & & $\Delta p$ & $N$ & & $p_{f}$ & & $\Delta p$ & $N$ \\
\hline 100 & (100) & 400 & 200 & 6 & 50 & $(50)$ & 200 & 200 & 6 \\
\hline 400 & (50) & 800 & 300 & 5 & 200 & (100) & 400 & 200 & 6 \\
\hline 800 & (25) & 1200 & 300 & 5 & 400 & (100) & 1000 & 300 & 5 \\
\hline 1200 & (100) & 1400 & 300 & 5 & 1000 & (200) & 1800 & 400 & 4 \\
\hline 1400 & (100) & 1800 & 400 & 4 & 1800 & (200) & 2000 & 500 & 3 \\
\hline \multirow[t]{2}{*}{1800} & (100) & 2500 & 500 & 3 & 2000 & (500) & 3000 & 500 & 3 \\
\hline & & & & & 3000 & $(500)$ & 5000 & 600 & 3 \\
\hline
\end{tabular}

(Table 5). The range and average GPE for each pressure is shown in Fig. 3. It is likely that the changes are associated with horizontal shifts of the flow field through the MODE region of a scale larger than the station grid or caused by inadequate spatial coverage to average out the effects of individual eddies.

\section{Error estimation}

In this section three questions about the APE calculation by different methods are discussed. First, which technique should be used in order to obtain a given accuracy of the estimate of APE? Second,

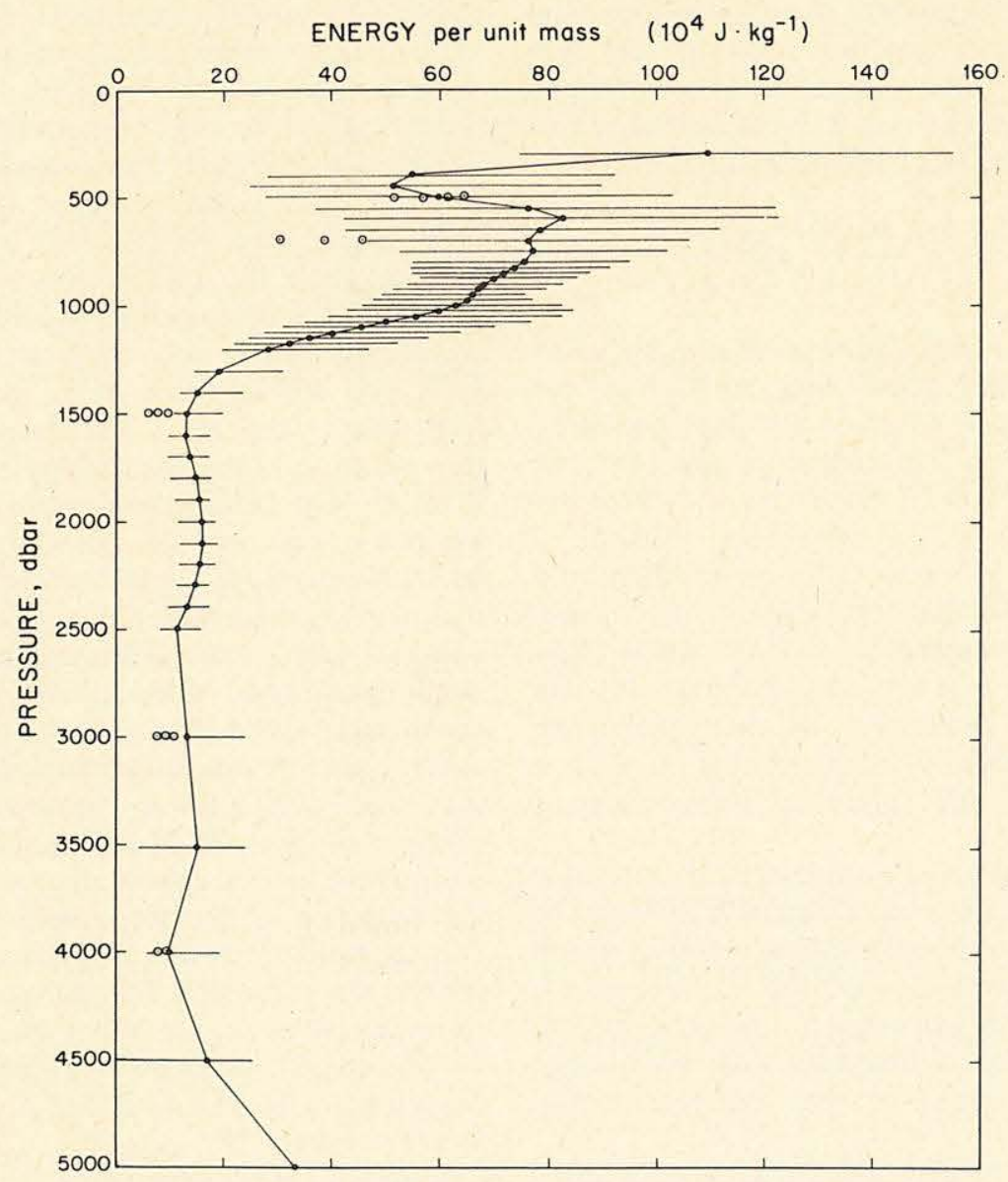

FIG. 3. Comparison of GPE and eddy kinetic energy per unit mass. Solid dots are GPE averaged over groups A-E, horizontal lines give the range over the five groups at each depth. Values above $2500 \mathrm{db}$ are taken from the high-resolution case, below 2500 from the low-resolution case (see Table 2). Open dots are eddy kinetic energy averaged over the duration of MODE (four months). 
what are the errors in APE which result from measurement errors and from finestructure variability? Finally, for a given set of observations, what is the sequence of steps which allows the optimization of either calculation?

In the following discussion several types of errors will be examined. Briefly, by measurement error is meant the uncertainty in the measured values of pressure, temperature and salinity (or conductivity) which results from uncorrected calibration errors (quantization noise is ignored) and which will be taken to be randomly distributed from one station to the next, but constant for a given station; random finestructure errors are the fluctuations in a derived variable caused by real variability of the field which occurs on smaller scales than those over which the calculations are performed; numerical integration errors are those errors which are a result of inadequate resolution of vertical structure and which may be reduced by decreasing the integration step size.

In this analysis errors in APE per unit mass and in APE per unit area are examined separately. Recall that TGPE and GPE represent the available gravitational potential energy per unit area and per unit mass, respectively. There are, in addition, the Boussinesq $\mathrm{APE}_{\mathrm{B}}$ (per unit mass) and the enthalpy formulation (HPE) of Lorenz (1955) and Reid et al. (1981):

$\mathrm{HPE}=\int_{p}^{p_{r}}\left(\bar{h}_{i}-h_{f}\right) d p^{\prime}=\frac{1}{2 g} \int_{p}^{p_{r}} \overline{\alpha_{p}^{*} \pi^{2}} d p^{\prime}+\mathrm{O}\left(\pi^{3}\right)$,

where $h_{i}$ and $h_{f}$ the initial and reference specific enthalpies. Two different Boussinesq calculations can be made, depending on whether the displacement is defined by the adiabatic leveling technique $(\pi)$ or by the difference between the observed and the mean specific volume $\left(\pi^{*}\right)$, i.e., $\pi^{*}=\left(\alpha_{i}-\bar{\alpha}\right) /\left(\alpha_{p}^{*}\right)$. If $\pi$ is small, there is very little quantitative difference between the two calculations of $\mathrm{APE}_{\mathrm{B}}$; however, for large displacements (as in Gulf Stream rings) the approximation $\pi=\pi^{*}$ may become invalid. Therefore, for the remainder of this discussion, $\mathrm{APE}_{\mathrm{B}}$ will be defined by $\mathrm{APE}_{\mathrm{B}}=1 / 2 \alpha_{p}^{*} \pi^{2}$, with $\pi$ the displacement of the reference steric anomaly field.

No exact expression for enthalpy in the ocean is available; therefore, the evaluation of HPE must be carried out in terms of the expansion about $\pi$, which requires higher order terms for large displacements. In what follows, HPE will refer to the evaluation of HPE to second order in displacement; notice that HPE is then equivalent to the vertical integral of $\mathrm{APE}_{\mathrm{B}}$.

Neither HPE nor TGPE is the "true" or net APE, and the amount of energy resulting from conversion of internal to potential energy which is actually available for conversion to kinetic energy is not known independently of the details of the conversions.
Therefore, the contributions to TGPE and GPE from changes in internal energy will be evaluated separately. Those terms are small compared to TGPE, as will be shown, and the error in estimating them for large displacements is also small. Thus, by subtracting those contributions from TGPE a minimum value of the net APE is obtained, nearly independent of errors which result from taking a finite number of terms in the expansion. The cumulative changes in internal energy in TGPE (Table 4) comprise 7\% of the total; the separate terms, which for this data set are of opposite sign, contribute roughly $12 \%$ (due to vertical gradients of compressibility) and $-5 \%$ (due to horizontal gradients). The comparable contributions to GPE are plotted as separate terms (for group C) in Fig. 4. The combined contribution to GPE is a maximum of $25 \%$, except for isolated shallow points where GPE falls to low values.

The integration step size is of some importance in making an accurate estimate of either TGPE or HPE, because of errors in the numerical integration over pressure. To examine the effects of integration errors two sets of parameters - low resolution (200 $\mathrm{m})$ and high resolution $(25 \mathrm{~m})$ of structure through the main thermocline-were applied to the MODE data. Table 2 lists the parameters in the two cases. TGPE and HPE were calculated for both cases for groups A-E. Large errors in the determination of both TGPE and HPE result from the inadequate resolution of the $200 \mathrm{~m}$ case (columns 7 and 8 of Table 3). The appropriate integration step sizes for a given application may be determined by comparing GPE and $A P E_{B}$, since $A P E_{B}$ is independent of the integration step size. The integration step size is decreased until the integration error is reduced sufficiently that GPE agrees with $\mathrm{APE}_{\mathrm{B}}$ to within the accuracy required. The compressibility effects may be included explicitly in the comparison by making use of the perturbation expansion (28). Using this comparison to reduce the integration error in GPE to some established value allows significant improvement in the estimates of both TGPE and HPE.

Random finestructure errors and measurement errors also contribute to the uncertainty in the calculation of APE. Both of these errors will be treated as random errors in this discussion; the values for the variance of pressure, temperature and salinity due to measurement error are taken from Scarlet (1974); the values for the variance of the regression estimates of pressure over each regression interval $\Delta p$ are calculated from the regression residuals, as described in Section 6. In the Appendix expressions are derived for the variance of TGPE and HPE as functions of the variance of the displacements $\pi_{k}$. The random finestructure and measurement errors in TGPE and HPE between 300 and $2500 \mathrm{db}$ are found in Table 4. Random errors in GPE for Group C were also calculated; they are presented in the form of 


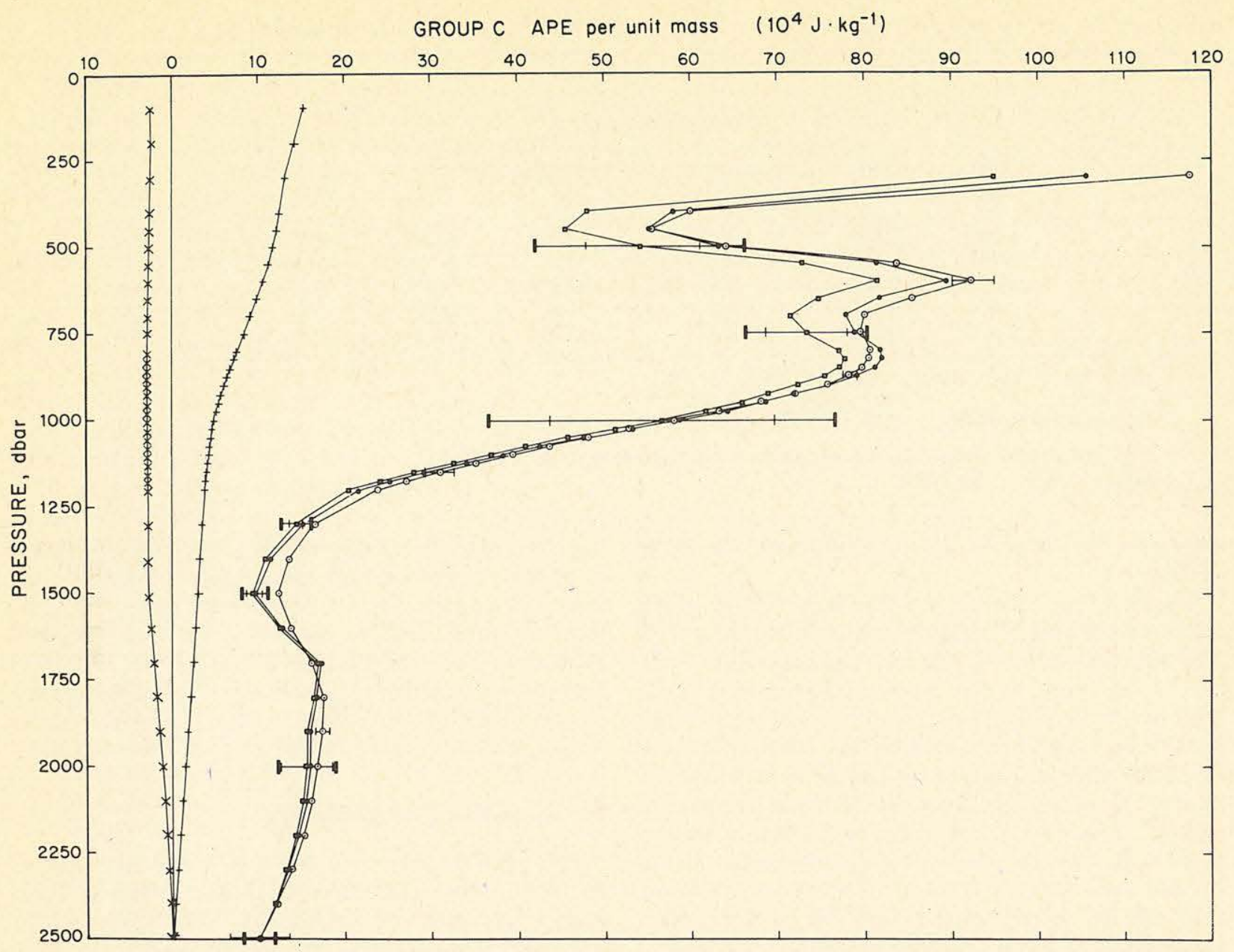

FIg. 4. APE per unit mass for group C. Open dots are GPE, squares are $A_{P E}$, and crosses and plus marks are the contributions from horizontal and vertical gradients of compressibility, respectively; solid dots are the sum of the Taylor expansion (28). Error bars with heavy end lines represent measurement errors, those with lighter lines represent finestructure errors.

error bars in Fig. 4. They are more strongly depth dependent and comprise a larger percentage than do the errors in TGPE.

Finally, in answer to the third question: from a practical viewpoint, it is of interest to establish a procedure for optimizing the calculation of both TGPE and HPE. A major part of that optimization involves the determination of adequate resolution of the vertical structure, as described earlier in this section; that procedure will not be discussed further, except to

TABLE 3. Comparison of TGPE and HPE between 300 and $2500 \mathrm{db}$ from low and high vertical resolution cases. Units are $10^{-4} \mathrm{~J}^{-2}$.

\begin{tabular}{|c|c|c|c|c|c|c|c|c|}
\hline \multirow[b]{2}{*}{ Group } & \multicolumn{3}{|c|}{ High resolution } & \multicolumn{3}{|c|}{ Low resolution } & \multicolumn{2}{|c|}{$\begin{array}{l}\text { Difference between } \\
\text { high and low }\end{array}$} \\
\hline & $\begin{array}{l}\text { TGPE } \\
\text { APE }\end{array}$ & $\begin{array}{l}\text { HPE } \\
\text { APE }\end{array}$ & $\Delta(\%)^{*}$ & $\begin{array}{l}\text { TGPE } \\
\text { APE }\end{array}$ & $\begin{array}{l}\text { HPE } \\
\text { APE }\end{array}$ & $\Delta(\%)^{*}$ & $\begin{array}{c}\Delta \text { TGPE } \\
(\%)^{* *}\end{array}$ & $\begin{array}{c}\Delta \mathrm{HPE} \\
(\%) \dagger\end{array}$ \\
\hline A & 0.746 & 0.660 & 11.6 & 1.022 & 1.094 & -7.0 & -37.0 & -65.8 \\
\hline B & 0.723 & 0.661 & 8.7 & 0.930 & 0.883 & 5.0 & -28.6 & -33.6 \\
\hline $\mathrm{C}$ & 0.836 & 0.752 & 10.0 & 0.846 & 0.637 & 24.6 & -1.2 & 15.3 \\
\hline D & 1.004 & 0.917 & 8.6 & 1.117 & 1.022 & 8.4 & -11.3 & -11.4 \\
\hline $\mathrm{E}$ & 0.746 & 0.661 & 11.5 & 0.925 & 0.835 & 8.9 & -23.9 & -26.4 \\
\hline
\end{tabular}

$* \Delta(\%)$ is (TGPE - HPE)/TGPE.

** $\Delta(\%)$ is [TGPE (high) - TGPE (low)]/TGPE (high).

$\dagger \Delta(\%)$ is [HPE (high) - HPE (low)]/HPE (high). 
TABLE 4. Errors in TGPE and HPE between 300 and $2500 \mathrm{db}$. Units are $10^{-4} \mathrm{~J} \mathrm{~m}^{-2}$.

\begin{tabular}{|c|c|c|c|c|c|c|c|c|}
\hline \multirow[b]{3}{*}{ Group } & \multicolumn{6}{|c|}{ Compressibility terms } & \multirow{2}{*}{\multicolumn{2}{|c|}{$\begin{array}{c}\text { Random } \\
\text { errors } \\
\text { finestructure } \\
\text { measurement }\end{array}$}} \\
\hline & TGPE & HPE & Horizontal* & Vertical** & & & & \\
\hline & APE & APE & $(\%)$ & $(\%)$ & $\sum \dagger$ & $\Delta(\%) \ddagger$ & $(\%)$ & $(\%)$ \\
\hline A & 0.746 & 0.660 & -4.1 & 14.9 & 0.740 & 0.8 & 1.2 & 4.5 \\
\hline B & 0.723 & 0.661 & -4.2 & 12.9 & 0.717 & 0.9 & 1.3 & 4.5 \\
\hline $\mathrm{C}$ & 0.836 & 0.752 & -5.5 & 12.3 & 0.809 & 3.2 & 0.6 & 3.3 \\
\hline D & 1.004 & 0.917 & -4.2 & 11.0 & 0.986 & 1.8 & 0.3 & 2.1 \\
\hline E & 0.746 & 0.661 & -4.5 & 12.7 & 0.772 & 3.3 & 0.8 & 3.8 \\
\hline
\end{tabular}

* Horizontal compressibility $=\int_{300}^{2500} g^{-1} \int_{p^{\prime}}^{2500} \overline{\kappa \pi} d p d p^{\prime}$.

** Vertical compressibility $=-\int_{300}^{2500} g^{-1} \int_{p^{\prime}}^{2500} 1 / 2 \overline{\Gamma_{\kappa} \pi^{2}} d p d p^{\prime}$.

$\dagger \sum$ is sum of HPE and horizontal and vertical compressibility terms [Eq. (40)].

$\ddagger \Delta(\%)$ is (TGPE $-\Sigma$ )/TGPE.

note where in the optimization sequence it should be performed.

The first step involves establishing the size of the interval over which the regressions will be performed. The interval must be larger than the maximum vertical displacement, so that a physically sensible result may be obtained from the inversion process. Since the displacement is not known beforehand, this can be an iterative procedure. A check on whether the interval is sufficiently large can be made by examining $\bar{\pi}$; as a rule of thumb, horizontal averages $\leqslant 10^{-2} \mathrm{db}$ indicate that the displacements do not exceed the intervals chosen. (For levels shallower than $300 \mathrm{~m}$, where density surfaces may intersect the surface of the ocean, the method is not valid.) The second step is the optimization of the polynomial order of the regression. The order should be high enough to resolve the vertical structure, but not so high that an instability of the regression occurs. (An instability is evidenced by large fluctuations of the regression coefficients.) The calculation is sensitive to changes in the polynomial order; for example, if the order chosen is too low by one, TGPE calculated will overestimate the correct value by $10-15 \%$. With the interval size and polynomial order decided, the integrations step sizes can be optimized as described earlier.

Finally, some type of weighting scheme must be used in the averaging process. Two schemes were tried on the MODE data: first, the weights derived from the objective mapping algorithm, as described in Section 7, and second, the simplest alternative of identical weights $\left(\omega_{k}=1\right)$ for each station. The difference in the APE per unit area between 300 and $2500 \mathrm{db}$ is less than $2 \%$; the differences in APE per unit mass are larger, but still less than the random errors. For Fig. 4, Tables 4 and 5, and the highresolution case in Table 3 the second scheme was used.

\section{Discussion and conclusions}

The MODE region, a $400 \mathrm{~km} \times 400 \mathrm{~km}$ square centered at $28^{\circ} \mathrm{N}, 69^{\circ} 40^{\prime} \mathrm{W}$, is characterized by lowenergy intensities (Dantzler, 1977; Schmitz, 1978) with eddies propagating westward (Freeland et al., 1976) and probably decaying (Bryden and Fofonoff, 1977). There is no clear evidence for local forcing or generation by baroclinic instability (MODE Group, 1978). Values obtained for APE per unit mass agree in magnitude and vertical distribution with estimates made by Kim (1975) using a Boussinesq approximation for two groups of 19 stations each. His two groups were composed of stations taken during March of 1973 (a subset of groups A and B) and June of 1973 (a subset of group E). Estimates of the average APE per unit mass for groups A-E and the range of values over the five groups (Fig. 3) indicate little storage of potential energy in the density structure. The values obtained are comparable to estimates of eddy kinetic energy (also, shown in Fig. 3)

TABLE 5. TGPE (between 300 and $2500 \mathrm{db}$ ) for each group compared with potential energy associated with changes in the reference specific volume anomaly $\delta_{f}$ from one group to another. Units are $10^{-4} \mathrm{~J} \mathrm{~m}^{-2}$.

\begin{tabular}{|c|c|c|c|c|c|c|}
\hline & \multicolumn{6}{|c|}{ Group } \\
\hline & A & B & $\mathrm{C}$ & D & $\mathrm{E}$ & A-E \\
\hline TGPE & 0.746 & 0.723 & 0.836 & 1.004 & 0.746 & \\
\hline Potential energy per unit area associated with changes in $\delta_{f}$ & \multicolumn{2}{|c|}{9.765} & & \multicolumn{2}{|c|}{2.894} & 21.337 \\
\hline
\end{tabular}



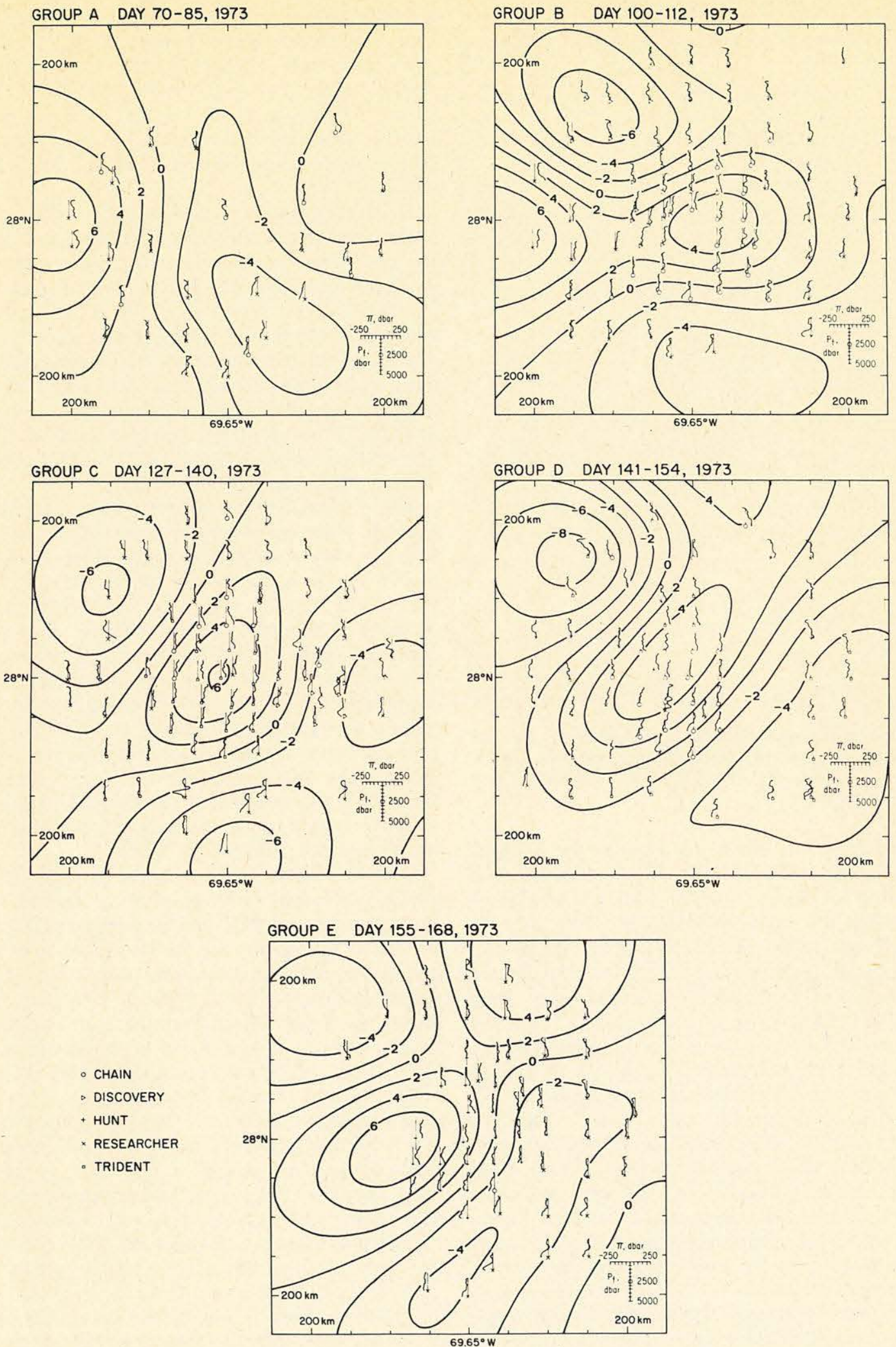

FIG. 5. Vertical profiles of the pressure displacements $\pi$ as a function of horizontal position for groups A to E. Downward displacements are positive. The dynamic heights at 500 relative to $2500 \mathrm{db}$ are superimposed to show the eddy structure. Dynamic height units are dynamic centimeters $\left(0.1 \mathrm{~J} \mathrm{~kg}^{-1}\right)$. 


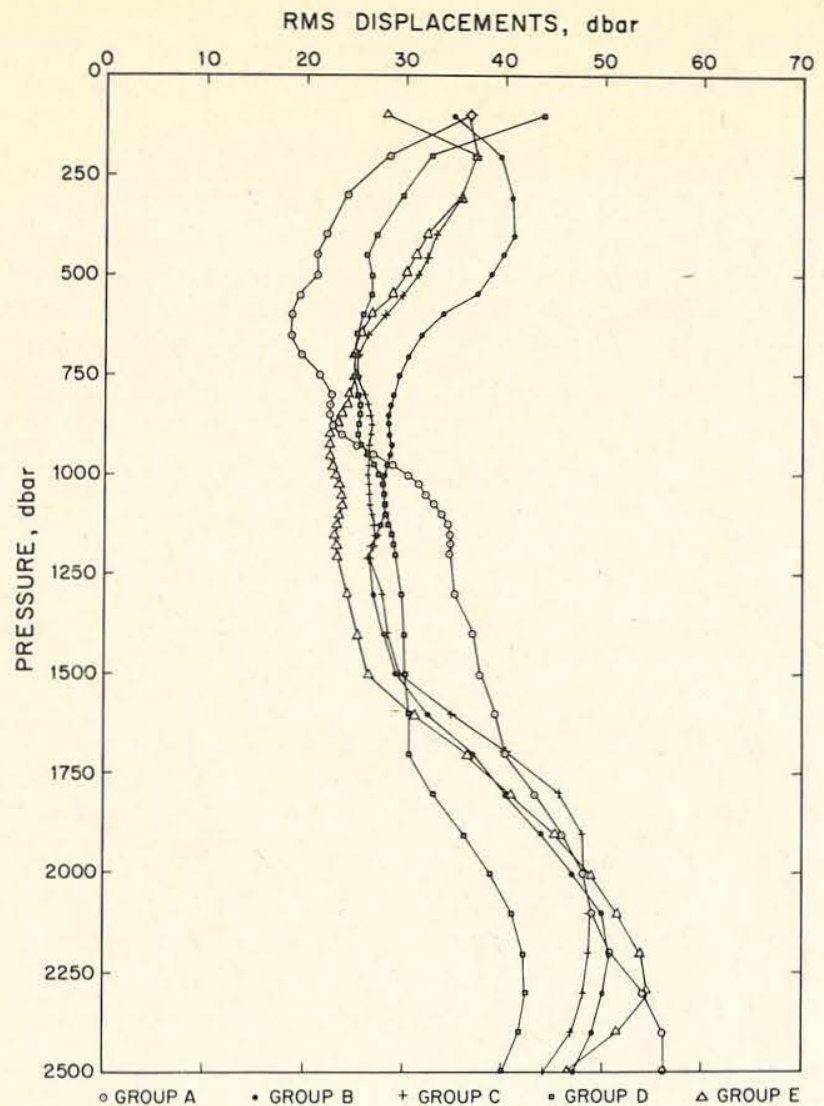

FIG. 6. Root-mean-square pressure displacements $\pi_{\text {rms }}$ of steric anomaly surfaces for groups A to E plotted against pressure. The displacements, which vary slowly with depth, reflect little influence of stratification.

calculated from current meter records by Schmitz (personal communication). Maximum values of APE per unit mass of about twice the eddy kinetic energy occur within the main thermocline. This may be compared with ratios of APE to kinetic energy of 3 and 2 estimated by Reid et al. (1981) for Gulf Stream rings sampled in 1967 and 1971, respectively. Reid et al. (1981) discuss the errors incurred when the far field density is used instead of the adiabatically leveled field as the reference for calculating APE in rings. The value obtained by Vulis and Monin (1975), converted to APE per unit mass, is $0.7 \mathrm{~J} \mathrm{~kg}^{-1}$ for the North Atlantic. Their estimate seems high, perhaps reflecting their neglect of the salinity field. Although reliable estimates of APE per unit mass could not be obtained in the near surface layers from the MODE data because density surfaces were not continuous over the entire region, the vertical average from the surface to $2500 \mathrm{db}$ is estimated to be about $0.006-0.010 \mathrm{~J} \mathrm{~kg}^{-1}$. Below $300 \mathrm{db}$ the average does not exceed $0.005 \mathrm{~J} \mathrm{~kg}^{-1}$. The low ratios of APE to eddy kinetic energy are interpreted as indicative of an inactive or decaying eddy regime. It seems unlikely that the baroclinic density field in the MODE region is the energy source for the eddies. The much larger changes of potential energy associated with changes of the reference density field from one Group to the next (Table 5) are indications of apparent shifts in the mean current flow with scales larger than the station grid, or of inadequate spatial coverage to average out the individual eddies.

Vertical displacements of density surfaces show a variety of structures with position and depth over the grid. The displacements are shown for groups A-E in Fig. 5, with the dynamic height at the $500 \mathrm{db}$ pressure surface relative to $2500 \mathrm{db}$ reference pressure superimposed to indicate the eddy structure. The westward propagation of the central anticyclonic eddy is evident. Downward displacements in the eddy of about $100 \mathrm{db}$ are nearly uniform in the upper $2500 \mathrm{db}$. These are particularly evident in group D. The upward displacements $(\pi<0)$ are less uniform with depth in the cyclonic eddies.

The vertical displacements do not reflect the stratification. Root-mean-square pressure displacements (Fig. 6) vary slowly with depth from about $35 \mathrm{db}$ at $300 \mathrm{db}$, to a broad minimum between 750 and $1250 \mathrm{db}$ of $\sim 25 \mathrm{db}$. Below $1250 \mathrm{db}$, there is a slow increase to roughly $50 \mathrm{db}$ at $2500 \mathrm{db}$. The increase continues below $2500 \mathrm{db}$ but the estimates are less reliable because of fewer stations and larger errors. It is surprising that the main thermocline at $800 \mathrm{db}$ affects the rms displacements so weakly. Because of the relative independence of amplitude of the displacements with depth, the horizontal density differences are controlled by the vertical stratification, which is largest at the main thermocline, producing the largest contribution to APE.

Over the entire depth interval studied (300-2500 $\mathrm{db})$ the horizontal variation of steric anomaly $\delta_{i}$ along each pressure surface can be estimated accurately by $-\alpha_{p}^{*} \pi$. This is not true for temperature or salinity below the main thermocline, indicating that considerable compensated horizontal structure exists in these two fields. The differences can be seen most clearly in plots of the ratios of the standard deviations of the initial and reference potential temperatures $\left(\left\langle\theta_{i}\right\rangle,\left\langle\theta_{f}\right\rangle\right)$ to the average temperature change $-\overline{d \theta / d p \pi_{\mathrm{rms}}}$ ( $\left(\right.$ implified to $-\bar{\theta}_{p} \pi_{\mathrm{rms}}$ ), shown in Fig. 7. If horizontal gradients along the reference steric surfaces $\delta_{f}$ were absent, $\left\langle\theta_{i}\right\rangle /\left(-\bar{\theta}_{p} \pi_{\text {rms }}\right)$ would be unity and $\left\langle\theta_{f}\right\rangle /\left(-\bar{\theta}_{p} \pi_{\text {rms }}\right)$ zero because adiabatic vertical displacements do not affect $\theta_{f}$. Horizontal structures in the reference surfaces would not be removed by leveling and would affect $\theta_{i}$ and $\theta_{f}$ equally if their vertical scale were larger than the displacement $\pi$. The effect is most noticeable between 1200 and 2000 $\mathrm{db}$, in the layer influenced by Mediterranean Water. Here the structure is dominated by horizontal differences.

The analysis presented here for a comparatively 


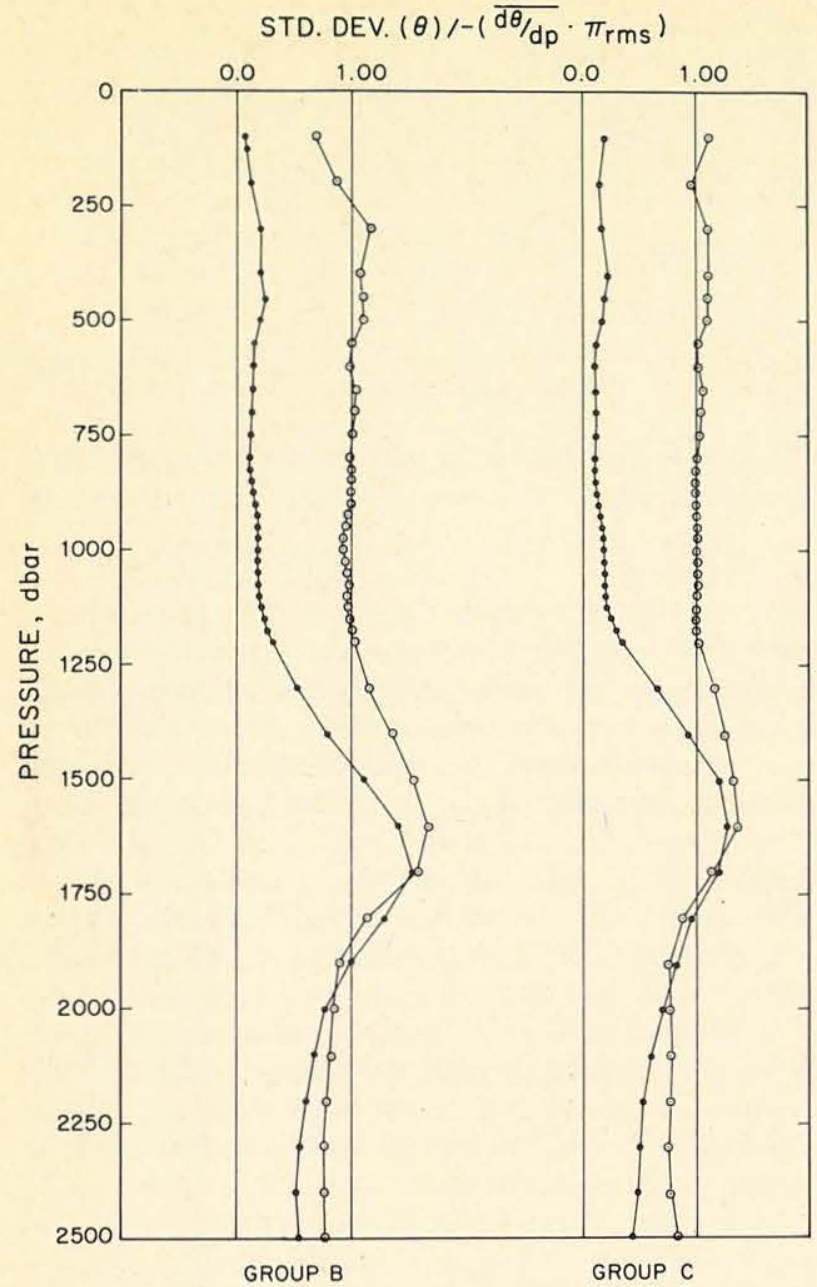

FIG. 7. Standard deviation of $\theta_{i}$ and $\theta_{f}\left(\left\langle\theta_{i}\right\rangle,\left\langle\theta_{f}\right\rangle\right)$ divided by $-\bar{\theta}_{p} \pi_{\text {rms }}$ for groups B and $\mathrm{C}$, showing the influence of horizontal variations of $\theta_{f}$ along the reference steric surfaces. Open dots are $\left\langle\theta_{i}\right\rangle /\left(-\bar{\theta}_{p} \pi_{\mathrm{rms}}\right)$, solid dots are $\left\langle\theta_{f}\right\rangle /\left(-\bar{\theta}_{p} \pi_{\mathrm{rms}}\right)$. Horizontal inhomogeneities of temperature dominate the observed temperature variations throughout the layer influenced by Mediterranean Water.

weak eddy field indicates that the available gravitational potential energy can be estimated to a precision of $15-30 \%$ by the simple Boussinesq approximation. The additional contributions from the nonlinearities of the equation of state tend to cancel, for the following reason. For a vertical displacement, if the compressibility increases downward, the leveling moves water of higher compressibility to higher pressure and lower compressibility to lower pressure giving a net decrease in volume and a positive contribution to GPE. In the horizontal structure the contribution is determined by the correlation between compressibility and displacement. For the MODE data the contribution to GPE is negative indicating that in regions of downward displacement (positive $\pi)$ the compressibility tends to be greater than in the upward displacements, yielding a net expansion of the layer on leveling and a negative contribution to GPE. As the compressibility depends primarily on temperature, the contributions can be of either sign depending on the vertical and horizontal variations of temperature. For small vertical displacements, the nonlinear terms can be estimated to adequate precision from the Taylor expansion in (28). For large vertical displacements such as are found in Gulf Stream rings, the local vertical gradient $\alpha_{p}^{*}$ is no longer a good estimate of the reference field gradient, the vertical displacement $\pi$ is no longer given satisfactorily by $\left(\alpha_{i}-\bar{\alpha}_{i}\right) /\left(\alpha_{p}^{*}\right)$ and the integral procedure should be used.

The algorithm for computing GPE also defines a set of adiabatically invariant steric surfaces over the station grid. These can be used to examine the distributions and changes of other conservative variables such as dissolved oxygen, silicates, etc. Vertical displacements of these surfaces should provide the most accurate estimates of changes in layer thickness used in vorticity balance calculations such as those made by McWilliams (1976).

Acknowledgment. We appreciated the opportunity to read a preprint of the paper by Reid, Elliott and Olson (1981) containing their comprehensive discussion of APE and TGPE for the atmosphere and ocean. As a result we were able to revise, correct and clarify a number of points in our manuscript prior to appearing in print. Although the basic formulations are essentially equivalent, the analytical treatment and applications are different and complementary.

This present work is supported by the Office of Naval Research under Contract N00014-76-C-197, NR 083-400.

\section{APPENDIX}

\section{Calculation of Systematic and Random Error in APE}

\section{Systematic differences between GPE and $A P E_{B}$}

The inclusion of compressibility effects in GPE was shown in Section 6 to introduce systematic differences between GPE and $\mathrm{APE}_{\mathrm{B}}$. Those differences were interpreted as contributions from horizontal and vertical gradients of compressibility, and it was noted that $-\overline{\alpha_{p}^{*} \pi}$ can be replaced by $\bar{\kappa} \pi$ in the horizontal term. It should also be noted that the computational algorithm calculates $\alpha_{p}^{*}\left(p_{i}\right)$ which differs from $\alpha_{p}^{*}\left(p_{f}\right)$. Differentiating (19) yields

$$
\begin{aligned}
\frac{d \alpha_{i}}{d p}\left(p_{i}\right)=\frac{d \alpha_{f}}{d p}\left(p_{f}\right)(1+ & \left.\frac{d \pi}{d p}\right) \\
& +\kappa\left(p_{i}\right)-\kappa\left(p_{f}\right)\left(1+\frac{d \pi}{d p}\right)
\end{aligned}
$$


or

$$
\alpha_{p}^{*}\left(p_{i}\right)=\alpha_{p}^{*}\left(p_{f}\right)\left(1+\frac{d \pi}{d p}\right) .
$$

Thus $\alpha_{p}^{*} \pi$ can be rewritten as

$\overline{\alpha_{p}^{*}\left(p_{f}\right) \pi}=\overline{\alpha_{p}^{*}\left(p_{i}\right) \pi}+\overline{\frac{d}{d p}\left(\frac{\alpha_{p}^{*} \pi^{2}}{2}\right)}-\overline{\frac{\pi^{2}}{2} \frac{d \alpha_{p}^{*}}{d p}}$.

This expansion may also be used to evaluate the contribution from horizontal gradients of compressibility; however, errors in the determination of the second derivative of $\alpha$ make it less satisfactory than the simpler $\kappa \pi$. The corresponding differences between TGPE and HPE are given by

horizontal gradients:

$$
\int_{p}^{p_{r}} g^{-1} \int_{p^{\prime}}^{p_{r}} \overline{\kappa \pi} d p^{\prime \prime} d p^{\prime}
$$

vertical gradients:

$$
-\int_{p}^{p_{r}} g^{-1} \int_{p}^{p_{r}} \frac{\overline{1 / 2 \Gamma_{\kappa} \pi^{2}}}{2} d p^{\prime \prime} d p^{\prime} .
$$

The contributions of these terms to TGPE between 300 and $2500 \mathrm{db}$ are given in Table 4; the contributions to GPE for Group C are plotted in Fig. 4.

\section{Random errors}

A general expression for the variance of HPE (defined as in Section 9) may be derived by assum- ing that all errors are uncorrelated from station to station and from level to level in the vertical, and that the variance of $\alpha_{p}^{*}$ is small compared to that of the displacement $\pi$. Then, in terms of sums over the integration steps $\Delta p$, the variance of HPE is

$$
\begin{aligned}
\mathrm{V}(\mathrm{HPE}) & =\mathrm{V}\left[\sum_{j=j(p)}^{j\left(p_{r}\right)}\left(\frac{1}{M} \sum_{k=1}^{M} \frac{1}{2 g} \alpha_{p}^{*} \pi_{k}^{2}\right)_{j} \Delta p_{j}\right] \\
& =\sum_{j=j(p)}^{j\left(p_{r}\right)}\left[\frac{1}{M} \sum_{k=1}^{M} \frac{\alpha_{p}^{* 2}}{4 g^{2}} \mathrm{~V}\left(\pi_{k}^{2}\right)\right]_{j} \Delta p_{j}{ }^{2} .
\end{aligned}
$$

Here $M$ is the number of stations and the subscript $j$ refers to the level. The variance of $\pi_{k}{ }^{2}$ is given by

$$
\mathrm{V}\left(\pi_{k}^{2}\right)=2\left[\mathrm{~V}\left(\pi_{k}\right)\right]^{2}+4\left\langle\pi_{k}\right\rangle^{2} \mathrm{~V}\left(\pi_{k}\right),
$$

with $\left\langle\pi_{k}\right\rangle$ the ensemble of mean of $\pi_{k}$, which is estimated by $\pi_{k}$ itself. The finestructure variance of $\pi_{k}$ is taken to be the variance of the regression estimate $p$, which is described in section 6 . (The variance of $\pi_{k}^{*}$, calculated from the mean field, may be larger than the variance of $\pi_{k}$, calculated from the adiabatically leveled reference field, since there is effectively less averaging in the determination of $\pi_{k}^{*}$.) The finestructure errors in HPE are found in Table 4.

Expressions for the variance of TGPE and GPE are most readily derived using the expansion of $\left(\alpha_{i}\right.$ $-\alpha_{f}$ ) from Eq. (18). (This is because $\alpha_{i}$ and $\alpha_{f}$ are obtained by inverting high order polynomials.) If any covariance between the terms in the perturbation expansion of TGPE are ignored, the variance of TGPE is then estimated as

$$
\begin{aligned}
\mathrm{V}(\mathrm{TGPE}) & =\mathrm{V}\left[\sum_{j=j(p)}^{j\left(p_{r}\right)} \frac{1}{g}\left\{\sum_{l=l(p)}^{l\left(p_{r}\right)}\left[\frac{1}{M} \sum_{k=1}^{M}\left(\alpha_{p}^{*} \pi+\frac{\Gamma_{k} \pi^{2}}{2}\right)_{l}\right] \Delta p_{l}\right] \Delta p_{j}\right\}+\mathrm{V}\left[\sum_{j=j(p)}^{j\left(p_{r}\right)} \frac{1}{2 g}\left(\frac{1}{M} \sum_{k=1}^{M} \alpha_{p}^{*} \pi^{2}\right) \Delta p_{j}\right] \\
& =\sum_{j=j(p)}^{j\left(p_{r}\right)}\left\{\frac{1}{M} \sum_{k=1}^{M} \frac{\alpha_{p}^{* 2}}{4 g^{2}} \mathrm{~V}\left(\pi_{k}^{2}\right)+\sum_{l=l(p)}^{l\left(p_{r}\right)} \frac{1}{g^{2}}\left[\frac{1}{M} \sum_{k=1}^{M} \frac{\Gamma_{k}{ }^{2}}{4} \mathrm{~V}\left(\pi_{k}{ }^{2}\right)+\mathrm{V}\left(\frac{1}{M} \sum_{k=1}^{M} \alpha_{p}^{*} \pi\right) \Delta p_{l}^{2}\right] \Delta p_{j}{ }^{2}\right\}, \quad(A
\end{aligned}
$$

with $\mathrm{V}\left(\pi_{k}{ }^{2}\right)$ given by (A3). The third term in (A4) requires some care in evaluation, because $\bar{\pi}$ is constrained to be zero. From Section 1 of the Appendix

$$
-\overline{\alpha_{p}^{*} \pi}=\overline{\kappa \pi}
$$

is very nearly constant; if it is taken to be a constant, the variance of $\overline{\kappa \pi}$ becomes

$$
V(\overline{\kappa \pi})=V(\kappa \bar{\pi})=\kappa^{2} V(\bar{\pi}) .
$$

In practice, $\bar{\pi}$ is not found to be exactly zero, due to numerical errors, so that some small contribution is to be expected from the third term. The variance of $\bar{\pi}$ is estimated as $(\bar{\pi})^{2}$. Values of the finestructure errors for TGPE are found in Table 4 and for GPE in Fig. 4. Because the finestructure errors in the compressibility terms are small, the error in $\mathrm{APE}_{\mathrm{B}}$ (using $\pi_{k}$ ) differs very little from that in GPE.
Measurement errors for GPE and TGPE were calculated (following Gregg, 1979) by assuming that only the pressure measurement error contributes to errors in $\pi_{k}$ (i.e., that temperature and salinity errors do not contribute) and that measurement errors in $\alpha_{p}^{*}$, and $\Gamma_{k}$ may be ignored. Scarlet's (1974) value for the pressure error ( $\sigma_{p}= \pm 4 \mathrm{db}$ ) was used as the standard deviation of $\pi_{k}$ in (A5). Contributions to the total measurement error from the error in the compressibility terms were found to be negligible; hence the measurement error in $\mathrm{APE}_{\mathrm{B}}$ is effectively the same as that calculated for GPE using (A4). The measurement error is plotted for GPE in Fig. 4, and tabulated for TGPE in Table 4.

\section{REFERENCES}

Barrett, J. R., 1971: Available potential energy of Gulf Stream rings. Deep-Sea Res., 18, 1221-1231. 
Bretherton, F. P., R. E. Davis and C. B. Fandry, 1976: A technique for objective analysis and design of oceanographic experiments applied to MODE-73. Deep-Sea Res., 23, 559582.

Brownlee, K. A., 1965: Statistical Theory and Methodology in Science and Engineering. Wiley, $590 \mathrm{pp}$.

Bryden, H., and N. P. Fofonoff, 1977: Horizontal divergence and vorticity estimates from velocity and temperature measurements in the MODE region. J. Phys. Oceanogr., 7, 329-337.

Cheney, R. E., and P. L. Richardson, 1976: Observed decay of a cyclonic Gulf Stream ring. Deep-Sea Res., 23, 143-145.

Dantzler, H. L., Jr., 1977: Potential energy maxima in the tropical and subtropical North Atlantic. J. Phys. Oceanogr., 1, 514519.

Fofonoff, N. P., 1962: Physical properties of sea water. The Sea: Ideas and Observations, Interscience, 3-50.

_ 1977: Computation of potential temperature of seawater for an arbitrary reference pressure. Deep-Sea Res., 24, 489491.

- , and H. L. Bryden, 1975: Specific gravity and density of seawater at atmospheric pressure. J. Mar. Res. (Suppl.), $33,69-82$.

- S. P. Hayes and R. C. Millard, Jr., 1974: WHOI/Brown CTD microprofiler: Methods of calibration and data handling. Woods Hole Oceanographic Institution Tech. Rep. WHOI $74-89,64 \mathrm{pp}$

Freeland, H., and J. Gould, 1976: Objective analysis of mesoscale ocean circulation features. Deep-Sea Res., 23, 915924.

Gandin, L. S., 1965: Objective analysis of meteorological fields. Israel Program for Scientific Translations. $242 \mathrm{pp}$. [NTIS TT-65-5000].

Gill, A. J., J. Green and A. Simmons, 1974: Energy partition in the large scale ocean circulation and the production of midocean eddies. Deep-Sea Res., 21, 499-528.

Gregg, M. C., 1979: The effects of bias error and system noise on parameters computed from $C, T, P$ and $V$ profiles. $J$. Phys. Oceanogr., 9, 199-217.

Holland, W. R., 1978: The role of mesoscale eddies in the general circulation of the ocean-numerical experiments using a wind-driven quasi-geostrophic model. J. Phys. Oceanogr., 8, 363-392.
Kim, K., 1975: Instability and energetics in a baroclinic ocean. Ph.D. thesis, MIT and WHOI Joint Program, 175 pp.

Lorenz, E. N., 1955: Available potential energy and the maintenance of the general circulation. Tellus, 2, 157-167.

Lynn, R. J., and J. L. Reid, 1968: Characteristics and circulation of deep and abyssal waters. Deep-Sea Res., 15, 577-598.

McWilliams, J. C., 1976: Maps from the Mid-Ocean Dynamics Experiment: Part II. Potential vorticity and its conservation. J. Phys. Oceanogr., 6, 828-846.

MODE Group, 1978: The Mid-Ocean Dynamics Experiment. Deep-Sea Res., 25, 859-910.

Montgomery, R. B., 1938: Circulation in upper layers of southern North Atlantic deduced with use of isentropic analysis. Pap. Phys. Oceanogr. Meteor., 6, 55 pp.

Peixóto, J. P., and A. H. Oort, 1974: The annual distribution of atmospheric energy on a planetary scale. J. Geophys. Res., 79, 2149-2159.

Reid, R. O., B. A. Elliott and D. B. Olson, 1981: Available potential energy: A clarification. J. Phys. Oceanogr., 11, $15-29$.

Rhines, P. B., 1977: The dynamics of unsteady currents. The Sea: Ideas and Observations on Progress in the Study of the Sea, Chap. 7, E. D. Goldberg, Ed., Wiley, 1048 pp.

Robinson, A. R., and J. C. McWilliams, 1974: The baroclinic instability of the open ocean. J. Phys. Oceanogr., 4, 281-294.

Rossby, C.-G., 1938: On the mutual adjustment of pressure and velocity distributions in certain simple current systems, II. J. Mar. Res., 1, 239-263.

Sandstrom, J. W. and B. Helland-Hansen, 1903: Ueber die Berechnung von Meereströmungen. Rep. Norweg. Fish Invest., 2, $43 \mathrm{pp}$.

Saunders, P., 1973: The instability of a baroclinic vortex. J. Phys. Oceanogr., 3, 61-65.

Scarlet, R. I., 1974: MODE-1 Density; Instrument Description and Intercomparison Report. MODE-1 Intercomparison Group, 93-96. 1975: A data processing method for salinity, temperature, depth profiles. Deep-Sea Res., 22, 509-515.

Schmitz, W. J., Jr., 1978: Observations of the vertical distribution of low frequency kinetic energy in the western North Atlantic. J. Mar. Res., 36, 295-310.

Vulis, I. L., and A. S. Monin, 1975: On the available potential energy of the ocean. Dokl. Akad. Nauk., 221, 597-600. 


\section{MANDATORY DISTRIBUTION LIST}

FOR UNCLASSIFIED TECHNICAL REPORTS, REPRINTS, AND FINAL REPORTS PUBLISHED BY OCEANOGRAPHIC CONTRACTORS

OF THE OCEAN SCIENCE AND TECHNOLOGY DIVISION

OF THE OFFICE OF NAVAL RESEARCH

(REVISED NOVEMBER 1978)

1 Deputy Under Secretary of Defense

(Research and Advanced Technology)

Military Assistant for Environmental Science Room 30129

Washington, D.C. 20301

Office of Naval Research

800 North Quincy Street

Arlington, VA 22217

3 ATTN: Code 483

1 ATTN: Code 460

2 ATTN: 102B

1 CDR J. C. Harlett, (USN)

ONR Representative

Woods Hole Oceanographic Inst.

Woods Hole, MA 02543

Commanding Officer

Naval Research Laboratory

Washington, D.C. 20375

6 ATTN: Library, Code 2627
12 Defense Documentation Center Cameron Station

Alexandria, VA 22314

ATTN: DCA

Commander Naval Oceanographic Office NSTL Station

Bay St. Louis, MS 39522

ATTN: Code 8100

ATTN: Code 6000

ATTN: Code 3300

1 NODC/NOAA

Code 0781

Wiscons in Avenue, N.W. Washington, D.C. 20235 
Approved for public release; distribution unlimited.

17. DISTRIDUTION STATEMENT (of the aboteact onfored in Block 20, il dillopent from Roport)

18. SUPPLEMENTARY NOTES

Reprinted from: Journal of Physical Oceanography 2(1): 30-47 (January 1981).

19. KEY WORDS (Contlnue on poverse alde if noceesery and ldentify by block number)

1. Available potential energy

2. Definition of: for the ocean physical oceanography

3. Observations of oceanic eddy energies.

20. ABSTRACT (Continue on roverse oldo if neceecery and Identtfy by block number)

See reverse side. 
20.

Available potential energy (APE) is defined as the difference between total potential plus internal energy of a fluid in a gravity field and a corresponding reference field in which the fluid is redistributed (leveled) adiabatically to have constant stably-stratified densities along geopotential surfaces. Potential energy changes result from local shifts of fluid mass relative to geopotential surfaces that are accompanied by local changes of enthalpy and internal energy and global shifs of mass (because volumes of fluid elements are not conserved) that do not change enthalpy or internal energy. The potential energy changes are examined separately by computing available gravitational potential energy (GPE) per unit mass and total GPE (TGPE) per unit area.

A technique for estimating GPE in the ocean is developed by introducing a reference density field (or an equivalent specific volume anomaly field) that is a function of pressure only and is connected to the observed field by adiabatic vertical displacements. The full empirical equation of state for seawater is used in the computational algorithm. The accuracy of the estimate is limited by the data and sampling and not by the algorithm itself, which can be made as precise as desired. 


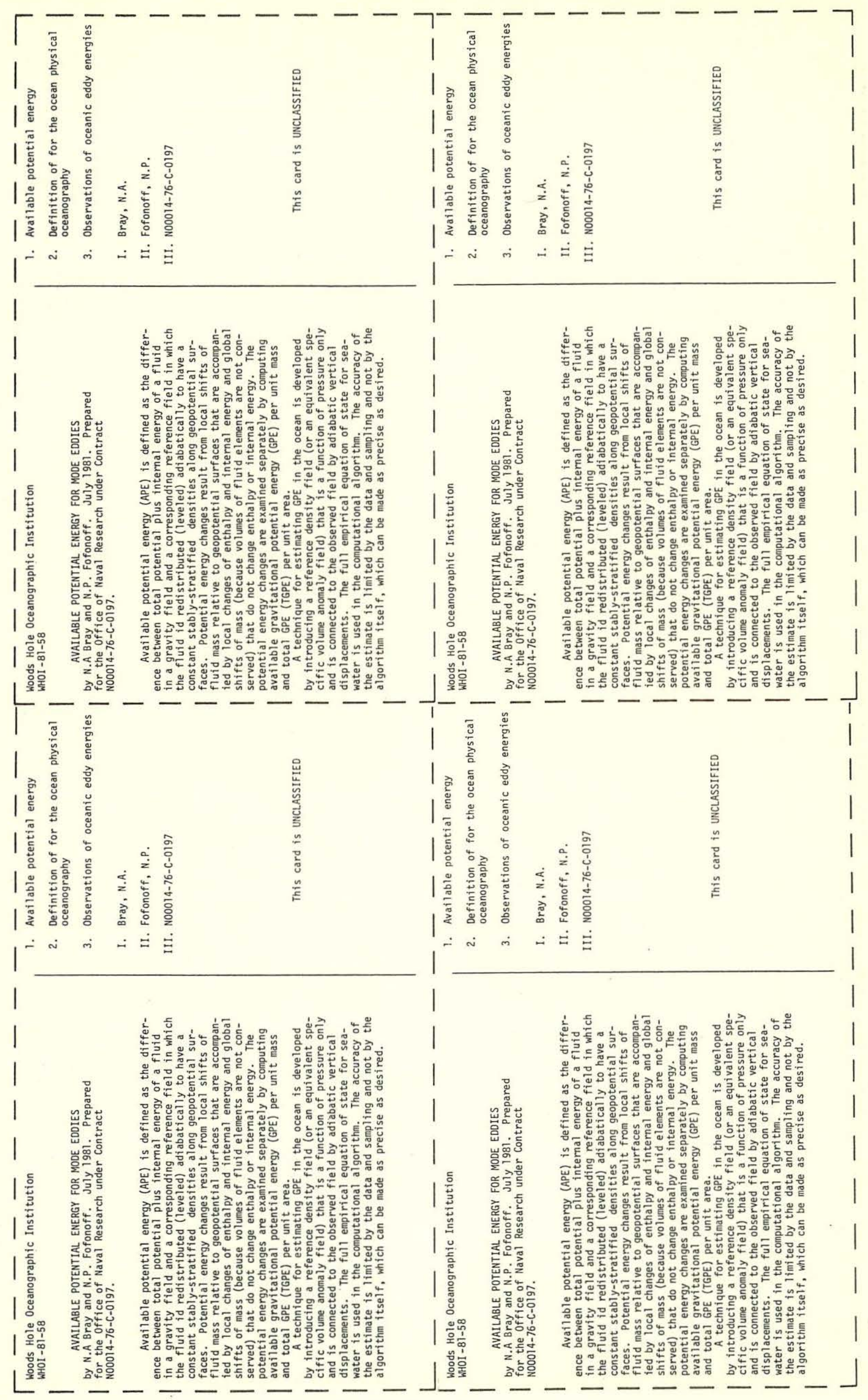

